\title{
The co-existence of hot and cold gas in debris discs ${ }^{\star}$
}

\author{
I. Rebollido ${ }^{1}$, C. Eiroa ${ }^{1}$, B. Montesinos ${ }^{2}$, J. Maldonado $^{3}$, E. Villaver ${ }^{1}$, O. Absil ${ }^{4}$, A. Bayo ${ }^{5,6}$, H. Canovas ${ }^{1,7}$, \\ A. Carmona ${ }^{8}$, Ch. Chen ${ }^{9}$, S. Ertel ${ }^{10}$, A. Garufi ${ }^{1}$, Th. Henning ${ }^{11}$, D. P. Iglesias ${ }^{5,6}$, R. Launhardt ${ }^{11}$, R. Liseau ${ }^{12}$, \\ G. Meeus ${ }^{1}$, A. Moor ${ }^{13}$, A. Mora ${ }^{14}$, J. Olofsson ${ }^{5,6}$, G. Rauw ${ }^{4}$, and P. Riviere-Marichalar ${ }^{15}$ \\ ${ }^{1}$ Departamento. Física Teórica, Universidad Autónoma de Madrid, Cantoblanco, 28049 Madrid, Spain \\ e-mail: isabel.rebollido@uam.es \\ ${ }^{2}$ Centro de Astrobiología (CAB, CSIC-INTA), ESAC Campus, Camino Bajo del Castillo s/n, Villanueva de la Cañada, 28692 Madrid, \\ Spain \\ 3 INAF, Osservatorio Astronomico di Palermo, Piazza del Parlamento 1, 90134 Palermo, Italy \\ ${ }^{4}$ STAR Institute, Université de Liège, F.R.S.-FNRS, 19c Allée du Six Août, 4000 Liège, Belgium \\ ${ }^{5}$ Instituto de Física y Astronomía, Facultad de Ciencias, Universidad de Valparaíso, Casilla, 5030 Valparaíso, Chile \\ ${ }^{6}$ Núcleo Milenio de Formación Planetaria-NPF, Universidad de Valparaíso, Av. Gran Bretaña, 1111 Valparaíso, Chile \\ ${ }^{7}$ European Space Astronomy Centre (ESA), PO Box 78, Villanueva de la Cañada, 28691 Madrid, Spain \\ ${ }^{8}$ Université de Toulouse, UPS-OMP, IRAP, 31400 Toulouse, France \\ ${ }^{9}$ Space Telescope Science Institute, 3700 San Martin Drive, Baltimore, MD 21212, USA \\ 10 Steward Observatory, Department of Astronomy, University of Arizona, Tucson, AZ 85721, USA \\ ${ }^{11}$ Max-Planck-Institut für Astronomie (MPIA), Königstuhl 17, 69117 Heidelberg, Germany \\ 12 Department of Space, Earth and Environment, Chalmers University of Technology, Onsala Space Observatory, 43992 Onsala, \\ Sweden \\ ${ }^{13}$ Konkoly Observatory, Research Centre for Astronomy and Earth Sciences, PO Box 67, 1525 Budapest, Hungary \\ ${ }_{14}$ Aurora Technology B.V. for ESA, ESA-ESAC, Villanueva de la Cañada, 28691 Madrid, Spain \\ 15 Instituto de Ciencia de Materiales de Madrid (CSIC), Calle Sor Juana Inés de la Cruz 3, 28049 Cantoblanco, Madrid, Spain
}

Received 20 November 2017 / Accepted 18 January 2018

\begin{abstract}
Context. Debris discs have often been described as gas-poor discs as the gas-to-dust ratio is expected to be considerably lower than in primordial, protoplanetary discs. However, recent observations have confirmed the presence of a non-negligible amount of cold gas in the circumstellar (CS) debris discs around young main-sequence stars. This cold gas has been suggested to be related to the outgassing of planetesimals and cometary-like objects.

Aims. The goal of this paper is to investigate the presence of hot gas in the immediate surroundings of the cold-gas-bearing debris-disc central stars.

Methods. High-resolution optical spectra of all currently known cold-gas-bearing debris-disc systems, with the exception of $\beta$ Pic and Fomalhaut, have been obtained from La Palma (Spain), La Silla (Chile), and La Luz (Mexico) observatories. To verify the presence of hot gas around the sample of stars, we have analysed the Ca II H\&K and the Na I D lines searching for non-photospheric absorptions of CS origin, usually attributed to cometary-like activity.

Results. Narrow, stable Ca II and/or Na I absorption features have been detected superimposed to the photospheric lines in 10 out of the 15 observed cold-gas-bearing debris-disc stars. Features are found at the radial velocity of the stars, or slightly blue- or red-shifted, and/or at the velocity of the local interstellar medium (ISM). Some stars also present transient variable events or absorptions extended towards red wavelengths (red wings). These are the first detections of such Ca II features in 7 out of the 15 observed stars. Although an ISM origin cannot categorically be excluded, the results suggest that the stable and variable absorptions arise from relatively hot gas located in the CS close-in environment of the stars. This hot gas is detected in at least $\sim 80 \%$, of edge-on cold-gas-bearing debris discs, while in only $\sim 10 \%$ of the discs seen close to face-on. We interpret this result as a geometrical effect, and suggest that the non-detection of hot gas absorptions in some face-on systems is due to the disc inclination and likely not to the absence of the hot-gas component. This gas is likely released in physical processes related in some way to the evaporation of exocomets, evaporation of dust grains, or grain-grain collisions close to the central star.
\end{abstract}

Key words. stars: general - planetary systems - comets: general - ISM: clouds - circumstellar matter

\section{Introduction}

Debris discs are second-generation dusty discs produced by collisions among planetesimals leftover from planet formation. They are observationally characterized by the thermal infrared

\footnotetext{
${ }^{\star}$ The reduced spectra are only available at the CDS (ascii files) and at the FEROS archive (FITS files) via anonymous ftp to cdsarc.u-strasbg.fr $(130.79 .128 .5)$ or via http://cdsarc.u-strasbg.fr/viz-bin/qcat?J/A+A/614/A3
}

excess observed in main-sequence stars (e.g. Backman \& Paresce 1993; Wyatt 2008, and references therein). Debris discs have been described as gas-poor discs, since in contrast with the "canonical" gas-to-dust ratio 100:1 in primordial discs, that ratio, although unconstrained, should be significantly lower (Liseau 2003; Matthews et al. 2014). However, following the first detection of CO in $\beta$ Pic (Vidal-Madjar et al. 1994; Roberge et al. 2000; Dent et al. 2014), and in 49 Ceti (Zuckerman et al. 1995; Hughes et al. 2008), a number of cold-gas-bearing debris-disc 
systems - currently 17 objects to our knowledge - have been detected through emission lines of O I, C I, C II and CO at farinfrared (FIR) and (sub-)millimetre wavelengths, mainly thanks to Herschel, APEX, JCMT, and ALMA (e.g. Moór et al. 2011, 2015a, 2017; Riviere-Marichalar et al. 2012, 2014; Roberge et al. 2013; Donaldson et al. 2013; Cataldi et al. 2014; Dent et al. 2014; Greaves et al. 2016; Lieman-Sifry et al. 2016; Marino et al. 2016). We note, nevertheless, that the $\mathrm{CO}$ emission associated with $\eta$ Crv requires confirmation (Marino et al. 2017). The gas in emission detected in these 17 stars is cold, $\mathrm{T} \sim 10-20 \mathrm{~K}$, and located at distances from tens to more than 100 au from the central star. It is also remarkable that numerous optical emission lines of $\mathrm{Ca}$ II, Na I, Fe I, Cr I+II, Ti I+II, and other metals, were detected towards the archetypical $\beta$ Pic debris disc (Olofsson et al. 2001; Brandeker et al. 2004). A detailed analysis of the optical data by those authors reveals that the optical atomic gas seen in emission is in Keplerian rotation and coexisting with the dust.

Stars with debris discs bearing cold gas tend to be young (ages $<50 \mathrm{Myr}$ ), but at least two of them, Fomalhaut and $\eta$ Crv, are significantly older; $\sim 400$ and $\sim 1400 \mathrm{Myr}$, respectively (Mamajek 2012; Marino et al. 2017). Most of them, 14 out of 17, surround A-type stars-the three remaining objects are F-type stars. All 17 systems have high fractional dust luminosities compared to other debris discs, $L_{\text {dust }} / L_{\star}>10^{-4}$ (e.g. Kennedy \& Wyatt 2014; Thureau et al. 2014; Moór et al. 2017, and references therein); and their spectral energy distributions (SEDs) have been fitted with two-temperature (warm and cold) components (e.g. Ballering et al. 2013; Melis et al. 2013; Chen et al. 2014; Kennedy \& Wyatt 2014; Thureau et al. 2014; Cotten \& Song 2016; Ballering et al. 2017). One exception is HD 172555, where only a warm component is present, at least up to the current detection limits (e.g. Riviere-Marichalar et al. 2012; Thureau et al. 2014).

The origin of the cold gas is a matter of debate. In the cases of HD 21997 and HD 131835 the gas could predominantly be a remnant of the primordial protoplanetary disc (Kóspál et al. 2013; Moór et al. 2015a). However, in most cases, for example, $\beta$ Pic or HD 181327 (Matrà et al. 2017; Marino et al. 2016), the observed gas most likely has a secondary origin. In primordial discs, $\mathrm{CO}$ is maintained against photodissociation as long as self-shielding is high enough to maintain a substantial CO mass, but the relatively low content of gas in the cold-gas-bearing debris discs suggests that self-shielding is low, and CO should be photodissociated on timescales of hundreds of years (van Dishoeck \& Black 1988; Roberge et al. 2000; Moór et al. 2011), requiring therefore a continuous replenishment to explain the observed emission in debris discs. A variety of physical processes have been proposed to explain the secondary origin of the cold gas, all of them in some way related to the presence of planetesimals-vaporization of solids in dust-dust collisions, photon-stimulated desorption of dust, collisions of volatile-rich dust grains, and outgassing of comet-like objects. Those mechanisms could even operate concurrently to explain the observed amount of gas (Matthews et al. 2014, and references therein).

In parallel, thanks to the favourable edge-on orientation of the $\beta$ Pic disc, stable and variable UV/optical absorption features in metallic lines have been known for several decades (Slettebak 1975; Kondo \& Bruhweiler 1985; Hobbs et al. 1985; Ferlet et al. 1987; Lagrange-Henri et al. 1988; Roberge et al. 2000). The variable, transient events, mainly traced in the Ca II K line ( $\lambda 3933.66 \AA$ ), appear red- and, to a much less degree, blue-shifted with respect to the radial velocity of the star, and might vary on timescales as short as hours. They have been interpreted as due to the gas released by the evaporation of exocomets grazing or falling onto the star, the "Falling Evaporating Bodies" scenario or FEBs (Ferlet et al. 1987; Beust et al. 1990; Kiefer et al. 2014b); Those FEBs would have been driven into the vicinity of the star by the perturbing action of a larger body, a planet (Beust et al. 1991; Beust \& Morbidelli 2000). Similar transient absorption events have been observed towards more than 20 A-type stars (e.g. Redfield et al. 2007; Roberge \& Weinberger 2008; Kiefer et al. 2014a; Welsh \& Montgomery 2015, 2018; Eiroa et al. 2016). Related to this, evidence of exocomet transits based on Kepler light curves of the F2 V stars KIC 3542116 and KIC 11083727 have recently been presented by Rappaport et al. (2018).

With respect to the stable absorption in $\beta$ Pic, in addition to the $\mathrm{Ca}$ II $\mathrm{H} \& \mathrm{~K}$ lines at the core of the photospheric lines that share the radial velocity of the star, similar absorption features appear in UV/optical lines of elements such as $\mathrm{C}, \mathrm{O}, \mathrm{Na}, \mathrm{Fe}$, and many other metallic species (Vidal-Madjar et al. 1994, 2017; Lagrange et al. 1998; Brandeker et al. 2004; Roberge et al. 2006). This gas would be located at distances of $\sim 0.5$ au, would be relatively hot with temperatures of $\sim 1000-2000 \mathrm{~K}$ (Hobbs et al. 1988; Beust et al. 1998; Vidal-Madjar et al. 2017), and could be generated by evaporation of comet-like bodies or dust grains in the disc (Fernández et al. 2006). However, this hot gas should rapidly be blown away by the strong radiation pressure from the star unless a braking mechanism is at work. Fernández et al. (2006) have suggested a solution to this problem, showing that the gas affected by the radiation pressure is largely ionized and couples into a single ionic fluid. As a consequence, the radiation pressure is reduced and the fluid could be self-braking if it has enhanced carbon abundance. Such a scenario is supported by the observed overabundance of $\mathrm{C}$ in $\beta$ Pic (Roberge et al. 2006; Brandeker 2011). A similar mechanism could be acting in the case of 49 Ceti (Roberge et al. 2014).

This work presents high-resolution optical spectra of the currently known cold-gas-bearing debris-disc stars. Our aim is to search for signposts of hot gas, which would suggest the presence of close-in cometary-like material around those stars. Thus, the analysis is mainly centered on the Ca II K ( $\lambda 3933.66 \AA)$ line as it is the most sensitive one to trace both transient and stable absorptions, but we also refer to the Ca II H $(\lambda 3968.47 \AA)$ and Na I D ( $\lambda \lambda$ 5889.95/5895.92 $\AA$ ) lines. The spectra have been obtained in the working frame of a large survey aiming at detecting and monitoring cometary-like absorption events in a sample of main-sequence stars, most of them A-type but also some FG-type (Rebollido et al., in prep.).

\section{Observations}

High-resolution optical spectra of 15 out of the currently known 17 cold-gas-bearing debris-disc stars were obtained with several fibre-fed high-resolution spectrographs and telescopes located at different observatories (Table 1). Namely, the spectrographs HERMES at Mercator and FIES at NOT (Roque de Los Muchachos, La Palma, Spain), FEROS at MPG/ESO $2.2 \mathrm{~m}$ (La Silla, Chile), and HEROS at the robotic telescope TIGRE (La Luz, Mexico). A series of observing runs were carried out at each telescope: Mercator (2015 September/December; 2016 January/ March/July; 2017 March/April), NOT (2016 January/July); MPG/ESO $2.2 \mathrm{~m}$ (2015 October; 2016 March; 2017 April); TIGRE (2015 September/November). The number of spectra per star and telescope is given in Table 1; a more detailed log with the specific dates (UT) of the observations of each star and the 
Table 1. Log of observations.

\begin{tabular}{|c|c|}
\hline Star & Number of spectra $(\mathrm{S} / \mathrm{N})$ \\
\hline HD 9672 & $31 \mathrm{H}(296)+7 \mathrm{FI}(161)$ \\
\hline HD 21997 & $13 \mathrm{H}(133)+3 \mathrm{FE}(281)+9 \mathrm{HE}(130)$ \\
\hline HD 32297 & 15 H (98), 4 FI (97) \\
\hline HD 109085 & $3 \mathrm{H}(85)$ \\
\hline HD 110058 & $8 \mathrm{FE}(280)$ \\
\hline HD 121191 & $1 \mathrm{FE}(90)$ \\
\hline HD 121617 & 2 FE (103) \\
\hline HD 131488 & $2 \mathrm{FE}(110)$ \\
\hline HD 131835 & $13 \mathrm{FE}(390)$ \\
\hline HD 138813 & $20 \mathrm{H}(205)+13 \mathrm{FE}(447)+4 \mathrm{FI}(102)$ \\
\hline HD 146897 & $2 \mathrm{H}(7)+4 \mathrm{FE}(112)$ \\
\hline HD 156623 & $13 \mathrm{FE}(314)$ \\
\hline HD 172555 & $17 \mathrm{FE}(380)$ \\
\hline HD 181296 & $16 \mathrm{FE}(373)$ \\
\hline HD 181327 & $5 \mathrm{FE}(241)$ \\
\hline
\end{tabular}

Notes. Number of spectra per instrument and signal-to-noise ratio (S/N) in brackets of the median spectra. H: HERMES; FE: FEROS; FI: FIES; HE: HEROS.

corresponding instrument is given in Table B. $1^{1}$. Spectral range and resolution of the different spectrographs are: HERMES, spectral resolution $\mathrm{R} \sim 85000$ covering the range $\lambda \lambda \sim 370$ $900 \mathrm{~nm}$ (Raskin et al. 2011); FIES, R 67000 covering $\lambda \lambda \sim 370-830 \mathrm{~nm}$ (Telting et al. 2014); FEROS, $\mathrm{R} \sim 48000$ and $\lambda \lambda \sim 350-929 \mathrm{~nm}$ (Kaufer et al. 1999); and HEROS, $\mathrm{R} \sim 20000$ and $\lambda \lambda \sim 350-880 \mathrm{~nm}$ (Schmitt et al. 2014). The spectra have been reduced using the available pipelines of the corresponding instrument; they include the usual steps for echelle spectra, such as bias substraction, flat-field correction, cosmic ray removal, and order extraction. Wavelength calibration is carried out by means of Th-Ar lamp spectra taken at the beginning and end of each night. Finally, barycentric corrections have been applied to all of them. Telluric lines heavily affect the wavelength range around the Na I D lines. To correct for them we have taken advantage of the fact that the spectra were obtained at many different epochs of the year. Thus, it is easy to identify and remove telluric lines, as they shift in wavelength when the barycentric correction is applied but the stellar $\mathrm{Na}$ I D lines remain at the same wavelength; furthermore, both lines of the $\mathrm{Na}$ I doublet share the same radial velocity. Table 1 also gives the signal-to-noise ratio of the median spectra achieved for each instrument in two "continua" at each side of the Ca II K line. Those values, and those of the individual spectra, are mostly affected by the observing weather conditions.

\section{Stellar properties of the observed sample}

Table 2 shows some of the observational and fundamental properties of the sample of stars presented in this work. Names, spectral types, and $B$ and $V$ magnitudes have been taken from SIMBAD (Wenger et al. 2000), while the distances are estimated from the revised HIPPARCOS parallaxes (van Leeuwen 2007) and/or the Gaia archive (Lindegren et al. 2016), except for HD 131488 taken from Melis et al. (2013). We note that there

\footnotetext{
1 The reduced spectra listed here are available at the CDS (ascii files) and at the FEROS archive (FITS files): http://archive.eso.org/wdb/wdb/adp/phase3_ spectral/form/orCDS
}

is a large discrepancy between the Gaia distance of HD 110058 of $188.7 \mathrm{pc}$ and the HiPPARCOS one of $107.4 \mathrm{pc}$. Ages from the literature and the corresponding references are also given in Table 2, as well as the list of papers reporting the cold gas detection.

$T_{\text {eff }}, \log g$, and $v \sin i$ have been estimated using the spectral synthesis programs ATLAS and SYNTHE (Kurucz 1993), fed with the models describing the stratification of the stellar atmospheres (Castelli \& Kurucz 2004). A grid of models with temperatures from 6000 to $10500 \mathrm{~K}$, step $100 \mathrm{~K}$, and $\log g=3.5$, 4.0, 4.5 was created and prepared to be broadened with any value of $v \sin i$. Solar abundances were used for all objects, with the exception of HD 32297 and HD 146897 as the fits clearly pointed towards a metallicity different from solar. For these two stars, metallicities $[\mathrm{Fe} / \mathrm{H}]-0.5$ and -0.2 were used, respectively (Gebran et al. 2016 give a value -0.7 for HD 32297).

For stars hotter than $\sim 8000 \mathrm{~K}$, the first step was to find the three models whose temperatures match the depth of the photospheric Ca II K line for the three values of $\log g$. The widths of $\mathrm{H} \gamma, \mathrm{H} \delta, \mathrm{H}[8-2]$ and $\mathrm{H}[9-2]$ were measured in the stellar spectra and in the models. Values of stellar gravities were found for each line by interpolating the width of the stellar lines in the models' measurements. The final value of the gravity, $\log g$, is the average of the individual determinations for each line, the uncertainty in the gravity being the standard error of the mean. The value of $\log g \pm \Delta \log g$ is then interpolated in the three models mentioned above to obtain the best fit value $T_{\text {eff }} \pm \Delta T_{\text {eff }}$. The uncertainties in $v \sin i$ have been estimated by comparing the width of the $\mathrm{Mg}$ II $4481 \AA$ absorption in the stellar spectrum with that of models computed with a range of rotation rates so as to allow the relative differences between star and model to be the order of $5 \%$.

For the F stars, the approach, being qualitatively similar, accounts for the different dependence of the calcium and hydrogen lines on temperature and gravity as compared with late-B or A-type stars. Once an acceptable model fitting the Ca II HK and the hydrogen lines is found, uncertainties are estimated by changing model parameters until the departures from the best fit are $\sim 10 \%$. The value of $v \sin i$ is estimated for this kind of stars by comparing the widths of weak photospheric lines with those of models and following a similar criterion as for hotter stars. Final typical errors are $\sim 100 \mathrm{~K}, \sim 0.20$, and $\sim 5 \mathrm{~km} \mathrm{~s}^{-1}$ for $T_{\text {eff }}$, $\log g$ and $v \sin i$, respectively.

\section{Results}

Narrow, stable, non-photospheric absorption features at the centre of, or slightly blue-/red-shifted with respect to, the photospheric Ca II K absorption line have been detected in 10 out of the 15 observed debris-disc systems (Table 3). The nonphotospheric absorption profile of some stars presents a red wing, suggesting that the observed profile is a blend of independent absorptions. In addition, a few stars show transient absorption events. In the following we describe the results of the stars with and without non-photospheric absorptions separately.

\subsection{Stars with non-photospheric Ca II absorptions}

Figure 1 shows the observed Ca II K line profiles of the observed stars with narrow, stable central absorptions, superimposed to the rotationally broadened photospheric line. The plotted, observed profile is the median of the spectra of each star. As far as we know, this is the first time that the $\mathrm{Ca}$ II $\mathrm{K}$ absorption feature is reported towards HD 32297, HD 131488, HD 131835 , HD 138813, HD 146897, HD 156623, and HD 181296 ( $\eta$ Tel). 
Table 2. Stellar properties of the observed cold-gas-bearing debris-disc stars.

\begin{tabular}{|c|c|c|c|c|c|c|c|c|c|c|}
\hline Star & Other name & SpT & $\begin{array}{l}d \\
(\mathrm{pc})\end{array}$ & $\begin{array}{l}V \\
(\mathrm{mag})\end{array}$ & $\begin{array}{l}B \\
\text { (mag) }\end{array}$ & $\begin{array}{l}T_{\text {eff }} \\
(\mathrm{K})\end{array}$ & $\log g$ & $\begin{array}{l}v \sin i \\
\left(\mathrm{~km} \mathrm{~s}^{-1}\right)\end{array}$ & $\begin{array}{l}\mathrm{Age}^{a} \\
(\mathrm{Myr})\end{array}$ & Cold gas detection ${ }^{b}$ \\
\hline HD 9672 & 49 Ceti & A1V & 59.4 & 5.61 & 5.67 & 9110 & 4.25 & 186 & $40(1)$ & (1) $\mathrm{CO}$ \\
\hline HD 21997 & HIP 16449 & $\mathrm{~A} 3 \mathrm{IV} / \mathrm{V}$ & 68.3 & 6.37 & 6.50 & 8610 & 4.10 & 60 & $42(2)$ & (2) $\mathrm{CO}$ \\
\hline HD 32297 & HIP 23451 & AOV & 136.2 & 8.14 & 8.32 & 7910 & 4.05 & 90 & $<30(3)$ & (3) $\mathrm{CO}$ \\
\hline HD 109085 & $\eta \mathrm{Crv}$ & F2V & 18.3 & 4.31 & 4.69 & 6950 & 4.20 & 60 & $1400(4)$ & (4) $\mathrm{CO}$ \\
\hline HD 110058 & HIP 61782 & A0V & 188.7 & 7.97 & 8.12 & 9720 & 4.45 & 150 & $15(5)$ & (5) $\mathrm{CO}$ \\
\hline HD 121191 & SAO 241295 & A5IV/V & 135.9 & 8.16 & 8.40 & 7970 & 4.25 & 65 & $16(5)$ & (6) $\mathrm{CO}$ \\
\hline HD 121617 & SAO 224570 & A1V & 128.2 & 7.29 & 7.36 & 9285 & 4.45 & 90 & $16(5)$ & (6) $\mathrm{CO}$ \\
\hline HD 131488 & SAO 320038 & A1V & 150.0 & 8.00 & 8.09 & 9050 & 4.21 & 120 & $16(5)$ & (6) $\mathrm{CO}$ \\
\hline HD 131835 & HIP 73145 & A2IV & 145.6 & 7.86 & 8.05 & 8610 & 4.35 & 105 & $16(5)$ & (7) $\mathrm{CO}$ \\
\hline HD 138813 & HIP 76310 & $\mathrm{~A} 0 \mathrm{~V}$ & 134.4 & 7.30 & 7.37 & 9685 & 4.45 & 130 & $10(5)$ & (5) $\mathrm{CO}$ \\
\hline HD 146897 & HIP 79977 & $\mathrm{~F} 2 / 3 \mathrm{~V}$ & 122.4 & 9.11 & 9.58 & 6700 & 4.30 & 55 & $15(6)$ & (5) $\mathrm{CO}$ \\
\hline HD 156623 & HIP 84881 & $\mathrm{~A} 0 \mathrm{~V}$ & 118.3 & 7.26 & 7.35 & 9580 & 4.10 & 80 & $16(5)$ & (5) $\mathrm{CO}$ \\
\hline HD 172555 & HIP 92024 & A7V & 28.6 & 4.77 & 4.97 & 7900 & 4.05 & 120 & $23(7)$ & (8) OI \\
\hline HD $181296^{c}$ & $\eta \mathrm{Tel}$ & $\mathrm{A} 0 \mathrm{~V}+\mathrm{M} 7$ & 48.2 & 5.02 & 5.04 & 10400 & 4.30 & 230 & $23(7)$ & (9) CII \\
\hline HD 181327 & HIP 95270 & F6V & 48.6 & 7.04 & 7.50 & 6650 & 4.40 & 28 & $23(7)$ & (10) $\mathrm{CO}$ \\
\hline
\end{tabular}

Notes. ${ }^{(a)}$ References for ages: (1) Torres et al. (2008), (2) Bell et al. (2015), (3) Kalas (2005), (4) Marino et al. (2017), (5) Pecaut \& Mamajek (2016), (6) Pecaut et al. (2012), (7) Mamajek \& Bell (2014). ${ }^{(b)}$ References for cold gas detection: (1) Zuckerman et al. (1995), (2) Moór et al. (2011), (3) Greaves et al. (2016), (4) Marino et al. (2017), (5) Lieman-Sifry et al. (2016), (6) Moór et al. (2017), (7) Moór et al. (2015a), (8) Riviere-Marichalar et al. (2012), (9) Riviere-Marichalar et al. (2014), (10) Marino et al. (2016) ${ }^{(c)}$ The M7 brown dwarf companion of $\eta$ Tel is located at an angular distance of 4" (Neuhäuser et al. 2011).

Table 3. Radial velocities and equivalent widths (EWs) of the Ca II H\&K and Na I D non-photospheric absorptions, radial velocities of the stars, and projected velocities of ISM clouds in the Colorado model.

\begin{tabular}{|c|c|c|c|c|c|c|c|}
\hline \multirow[b]{2}{*}{ Star } & \multicolumn{5}{|c|}{ Stars with non-photospheric absorptions } & \multirow[b]{2}{*}{$\begin{array}{l}\mathrm{v}_{\text {rad }} \\
\left(\mathrm{km} \mathrm{s}^{-1}\right)\end{array}$} & \multirow[b]{2}{*}{$\begin{array}{r}\mathrm{V}_{\mathrm{ISM}}{ }^{d} \\
\left(\mathrm{~km} \mathrm{~s}^{-1}\right)\end{array}$} \\
\hline & $\begin{array}{r}\mathrm{v}_{\text {CaIIK }} \\
\left(\mathrm{km} \mathrm{s}^{-1}\right)\end{array}$ & $\begin{array}{c}\mathrm{EW}(\mathrm{K} / \mathrm{H}) \\
\mathrm{m \AA}\end{array}$ & $\begin{array}{r}\Delta \mathrm{v}_{\text {CaIIK }} \\
\left(\mathrm{km} \mathrm{s}^{-1}\right)\end{array}$ & $\begin{array}{c}\mathrm{v}_{\mathrm{NaID}} \\
\left(\mathrm{km} \mathrm{s}^{-1}\right)\end{array}$ & $\begin{array}{c}\mathrm{EW}(\mathrm{D} 2 / \mathrm{D} 1)^{c} \\
\mathrm{m \AA}\end{array}$ & & \\
\hline HD $9672^{a}$ & $13.5 \pm 2.3$ & $10.6 / 6.0$ & 7.4 & - & $<1.5$ & $11.0 \pm 2.0$ & $11.01(\mathrm{LIC})$ \\
\hline HD $32297^{a}$ & $22.7 \pm 3.0$ & $19.4 / 8.9$ & 9.7 & $23.8 \pm 2.5$ & $93.0 / 84.0$ & $19.7 \pm 2.0$ & 23.59 (LIC) \\
\hline \multirow[t]{2}{*}{ HD 110058} & $12.7 \pm 3.3$ & $27.0 / 24.1$ & 6.8 & $12.5 \pm 2.8$ & $46.0 / 39.1$ & $12.6 \pm 2.4$ & $-14.46(\mathrm{G})$ \\
\hline & $1.2 \pm 3.3$ & $19.9 / 10.0$ & 12.6 & $0.9 \pm 2.8$ & $34.0 / 18.9$ & & \\
\hline \multirow[t]{2}{*}{ HD 131488} & $4.0 \pm 2.7$ & $5.7 /-$ & 9.6 & $5.3 \pm 2.2$ & $42.1 / 34.2$ & $5.8 \pm 1.5$ & $-23.91(\mathrm{G})$ \\
\hline & $-3.2 \pm 2.7$ & $4.0 /-$ & 4.2 & - & $<1.6$ & & \\
\hline \multirow[t]{3}{*}{ HD 131835} & $2.2 \pm 2.7$ & $0.7 /-$ & 2.3 & - & $<0.9$ & $2.6 \pm 1.4$ & $-18.56(\mathrm{GEM})$ \\
\hline & $-5.9 \pm 2.7$ & $2.1 / 1.4$ & 7.9 & - & $<0.9$ & & \\
\hline & $-13.8 \pm 2.7$ & $8.3 / 4.1$ & 7.4 & $-11.8 \pm 2.0$ & $41.0 / 32.1$ & & \\
\hline HD 138813 & $-27.5 \pm 3.0$ & $5.6 /-$ & 18.7 & $-12.0 \pm 2.5$ & $18.0 / 15.4$ & $-0.4 \pm 2.0$ & $-27.82(\mathrm{G})$ \\
\hline \multirow[t]{2}{*}{ HD 146897} & $-8.0 \pm 2.7$ & $12.4 /-$ & 7.2 & $-8.8 \pm 2.1$ & $118.2 / 80.0$ & $-4.0 \pm 1.5$ & $-29.19(\mathrm{G})$ \\
\hline & $-30.0 \pm 2.7$ & 5.9/- & 4.6 & - & $<0.8$ & & \\
\hline \multirow[t]{2}{*}{ HD $156623^{a}$} & $-15.0 \pm 2.7$ & $9.0 / 3.7$ & 18.7 & - & $<0.7$ & $-0.2 \pm 1.5$ & $-24.89(\mathrm{G})$ \\
\hline & $8.1 \pm 2.7$ & $4.3 /-$ & $6.0^{e}$ & - & $<0.7$ & & \\
\hline HD $172555^{a}$ & $2.3 \pm 3.0$ & $9.9 / 4.0$ & 9.7 & - & $<0.7$ & $2.6 \pm 1.9$ & $-16.84(\mathrm{G})$ \\
\hline \multirow[t]{2}{*}{ HD $181296^{b}$} & $-22.8 \pm 3.8$ & $3.9 / 2.2$ & 8.4 & - & $<0.7$ & $-3.0 \pm 3.0$ & $-18.51(\mathrm{G})$ \\
\hline & \multicolumn{5}{|c|}{ Stars without non-photospheric absorptions } & & \\
\hline HD 21997 & - & - & - & - & - & $17.3 \pm 2.0$ & $15.87(\mathrm{LIC})$ \\
\hline HD 109085 & - & - & - & - & - & $-0.4 \pm 1.0$ & -0.11 (GEM) \\
\hline HD 121191 & - & - & - & - & - & $12.0 \pm 1.4$ & $-18.34(\mathrm{G})$ \\
\hline HD 121617 & - & - & - & - & - & $7.8 \pm 1.6$ & $-19.84(\mathrm{G})$ \\
\hline HD $181327^{b}$ & - & - & - & - & - & $0.2 \pm 1.5$ & $-18.51(\mathrm{G})$ \\
\hline
\end{tabular}

Notes. The FWHM velocity dispersion of the Ca II K lines is also given. ${ }^{(a)}$ Stars with FEBs. ${ }^{(b)}$ The line of sight towards these two stars does not traverse any ISM cloud in the Colorado model but it passes within $20^{\circ}$ of several clouds. Among these, cloud $\mathrm{G}$ is the closest one in velocity to these stars. ${ }^{(c)}$ Upper limits are shown for those stars without Na non-photospheric absorption. ${ }^{(d)}$ Names within parentheses are ISM clouds in the Colorado model (Redfield \& Linsky 2008). ${ }^{(e)}$ The velocity dispersion for this absorption is highly variable, and the value shown here is the value obtained from the median spectra. 


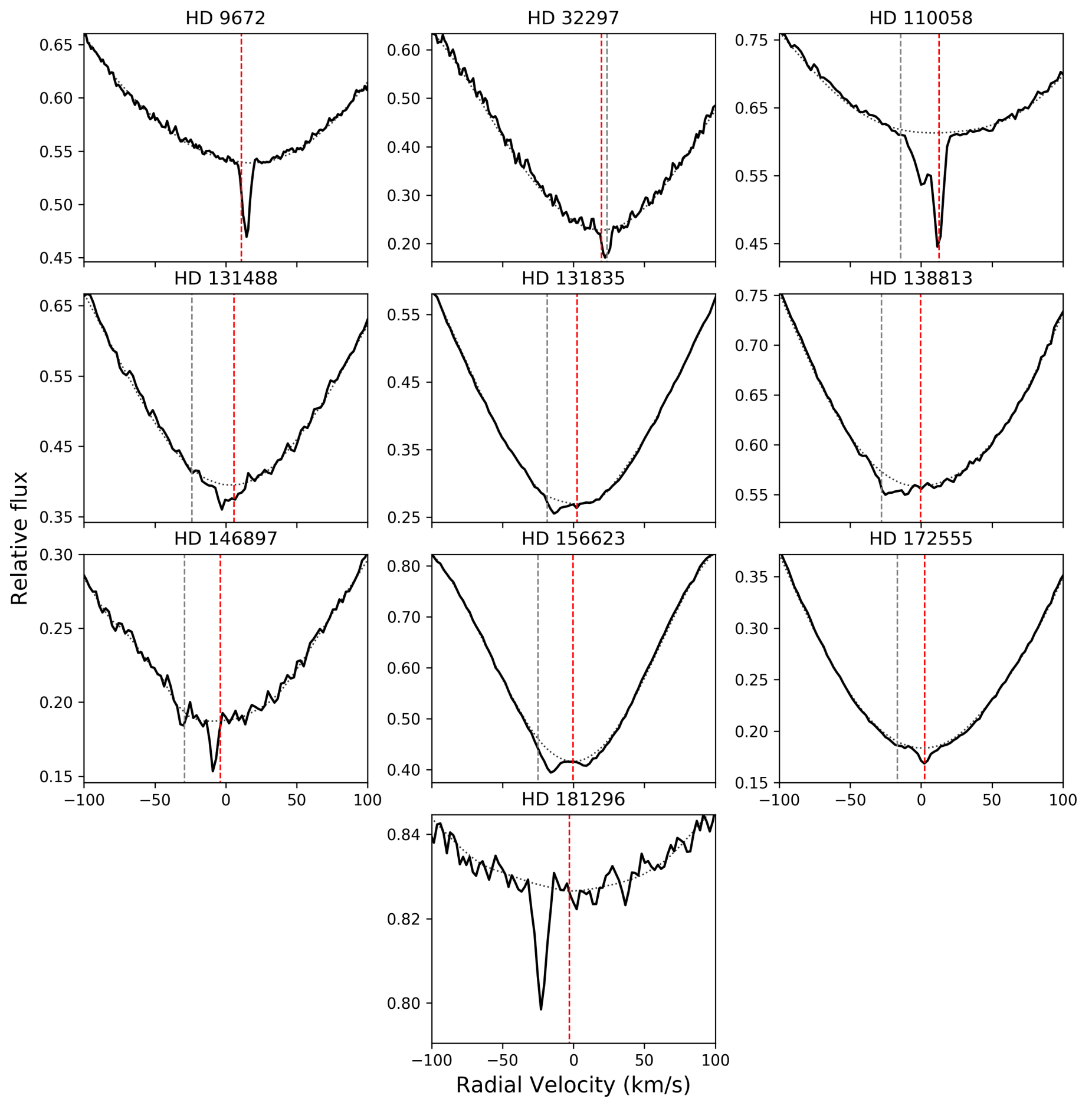

Fig. 1. Median Ca II K line profiles of the gas-bearing debris-disc stars with non-photospheric absorption (black solid line) together with a Kurucz photospheric model (black dotted line) with stellar parameters according to Table 2. Dashed lines in all panels indicate the corresponding radial velocities of the stars (red), and the projected velocities of the local ISM (grey) after the Colorado model (Redfield \& Linsky 2008). Velocities are in the reference frame of the Ca II K line. $v_{\text {rad }}$ and $v_{\text {ISM }}$ are coincident for HD 9672 (see Table 3). HD 181296 has no ISM lines in the line of sight (see Table 3 and Sect. 5.2 for a discussion). The same convention for $v_{\text {rad }}$ and $v_{\text {ISM }}$ is used in all further figures.

Welsh \& Montgomery (2018) have also recently detected the narrow absorption towards $\eta$ Tel. Previous narrow absorption detections have been published in the cases of 49 Ceti, HD 110058, and HD 172555 (Montgomery \& Welsh 2012; Kiefer et al. 2014a; Hales et al. 2017). Table 3 gives equivalent widths (EWs), radial velocities, and velocity dispersion (full width at half maximum (FWHM)) of all non-photospheric Ca II K features. The table also gives EWs of the $\mathrm{Ca}$ II $\mathrm{H}$ line in those cases where the line can be distinguished from the strong $\mathrm{H} \epsilon$ Balmer line, as well as
EWs and velocities of the $\mathrm{Na}$ I $\mathrm{D}$ lines when detected. In the case of non-detection in Na I, upper limits of the D2 line (the strongest one of the doublet) were calculated as EW of Gaussians with parameters determined by the instrumental resolution (width) and the $3 \sigma$ rms noise in the wavelength region (depth). In case of detection, EWs are the average of independent measurements made with three different software packages-MIDAS, dipso and Gaussian fits made with Python. Estimated uncertainties are of the order of $10 \%$. Velocity dispersions in Table 3 were 
calculated in the median spectra as the FWHM of fitted Gaussians. Radial velocities were measured as the central value of the non-photospheric absorption. Uncertainties were estimated as the square sum of the uncertainties in the radial velocity of the star (see below) and the size of the pixel in velocity.

Stable absorptions might originate in CS discs sharing the radial velocity of the central star, or in the complex local interstellar medium (ISM) (e.g. Redfield \& Linsky 2008). In order to try to decipher which of these alternatives corresponds to reality, radial velocities of the stars were estimated using the Balmer lines and the velocity shift of the Kurucz models to fit the observed Ca II K photospheric line (Table 3). Quoted $v_{\text {rad }}$ errors correspond to the difference between both methods. For the sake of completeness, radial velocities of the stars and of the corresponding ISM velocity vectors are also given in Table 3 for those stars without non-photospheric absorptions. Our stellar radial velocity estimates are in good agreement with previous ones (e.g. de Bruijne \& Eilers 2012; Melis et al. 2013; Hales et al. 2017). For the ISM velocity vectors, we have used Redfield \& Linsky's Colorado model ${ }^{2}$ (Redfield \& Linsky 2008) to estimate them along the line of sight towards the stars. Names of the corresponding ISM clouds are also indicated in Table 3. In the cases of HD 181296 and HD 181327, their lines of sight do not traverse any cloud in the Colorado model, although they pass within $20^{\circ}$ of several clouds. A complementary search of ISM absorption features has been conducted in the cases of HD 131488, HD 131835, HD 138813, and HD 146897. This search was motivated since, based on previous results and on our own spectra, the origin of the observed non-photospheric absorptions was highly ambiguous. In this context, we have analysed the $\mathrm{Ca}$ II and $\mathrm{Na}$ I lines of field stars surrounding those four coldgas-bearing debris discs. We note that these stars are located in the line of sight of the G or Gem Colorado clouds (Table 3), and are members of the Scorpius-Centaurus OB association (Pöppel et al. 2010; Lieman-Sifry et al. 2016). Details of this search are given in Appendix A. The remaining 11 objects in the sample were excluded from this study due either to the presence of variable non-photospheric absorptions, or the unambiguous determination of a CS absorption.

\section{Individual stars}

HD 9672 (49 Ceti). A Ca II K narrow, stable absorption at the bottom of the photospheric line at $\sim 13.5 \mathrm{~km} \mathrm{~s}^{-1}$ and EW(Ca II K) $\sim 10.6 \mathrm{~m} \AA$ is detected. This feature coincides with the radial velocities of the star and of the local ISM (Fig. 1 and Table 3). Our result is similar to the stable component observed by Montgomery \& Welsh (2012). Using the high-resolution spectrograph STIS at the HST, Malamut et al. (2014) detected two partially blended, near-UV Mg II and Fe II features with velocities 9.0 and $14.4 \mathrm{~km} \mathrm{~s}^{-1}(\mathrm{Mg} \mathrm{II})$, and 11.0 and $13.65 \mathrm{~km} \mathrm{~s}^{-1}$ (Fe II), and suggested the second one could be attributed to the CS disc. Thus, it is most likely that the absorption we detect is a blend of both the CS and the ISM components. The $\mathrm{Ca}$ II $\mathrm{H}$ line is detected with $\mathrm{EW}(\mathrm{Ca}$ II $) \sim 6.0 \mathrm{~m} \AA$, which suggests that the $\mathrm{Ca}$ II lines are optically thin. None of our spectra show any transient Ca II absorption in contrast with the FEBs detected by Montgomery \& Welsh (2012). Neither stable components nor any variable features are detected in the $\mathrm{Na}$ I D lines.

$H D$ 32297. The radial velocity of the $\operatorname{star}\left(19.7 \mathrm{~km} \mathrm{~s}^{-1}\right)$ is very close to that of the local ISM. The velocity and EW of the detected Ca II K stable absorption is $\sim 22.7 \mathrm{~km} \mathrm{~s}^{-1}$ and

2 http://sredfield.web.wesleyan.edu/ $\sim 19.4 \mathrm{~m} \AA$, respectively (Fig. 1 and Table 3 ). The $\mathrm{Ca}$ II $\mathrm{H}$ line is detected with $\mathrm{EW}(\mathrm{Ca}$ II H) $\sim 8.9 \mathrm{~m} \AA$. Very high-resolution spectra, $\mathrm{R} \sim 240000$, of the $\mathrm{Na}$ I doublet show two components at $\sim 20.5 \mathrm{~km} \mathrm{~s}^{-1}$ and $\sim 24.5 \mathrm{~km} \mathrm{~s}^{-1}$, attributed to the CS medium and the ISM, respectively (Redfield et al. 2007). This suggests again that the $\mathrm{Ca}$ II absorptions we detect are a blend of both CS and ISM components. Redfield et al. (2007) detected variability in the Na I lines; in our case, no apparent variability is detected either in the $\mathrm{Ca}$ II lines or in the $\mathrm{Na} I$ ones.

$H D$ 110058. Two Ca II K components at velocities of 12.7 and $1.2 \mathrm{~km} \mathrm{~s}^{-1}$ are detected with EW $\sim 27 \mathrm{m \AA}$ and $\sim 20 \mathrm{m \AA}$, respectively (Fig. 1 and Table 3). The $12.7 \mathrm{~km} \mathrm{~s}^{-1}$ absorption coincides with the radial velocity of the star, while the ISM velocity towards this line of sight is $-14.46 \mathrm{~km} \mathrm{~s}^{-1}$. The same velocity components have been reported by Hales et al. (2017) and Iglesias et al. (in prep.). However, while both features remain stable in our spectra (taken in the same campaign), a comparison with those of similar spectral resolution (Hales et al. 2017) suggests that the strength of the blueshifted component varies. Hales et al. (2017) ascribe this absorption to the ISM because of the presence of a similar feature in the spectra of close-by stars. However, the fact that its strength might vary suggests that it might well have a CS origin. Further monitoring of this star is required to elucidate this apparent controversy. Both features are detected in the $\mathrm{Ca}$ II $\mathrm{H}$ line with $\mathrm{EW} \sim 24.0$ and $\sim 10.0 \mathrm{~m} \AA$, respectively. This result suggests that the stronger $12.7 \mathrm{~km} \mathrm{~s}^{-1}$ absorption is optically thick, while the weaker one at $1.2 \mathrm{~km} \mathrm{~s}^{-1}$ is optically thin. A similar behaviour is present in the $\mathrm{Na} \mathrm{I} \mathrm{D}$ lines (Table 3, Hales et al. 2017; Iglesias et al., in prep.).

HD 131488. The non-photospheric Ca II K profile shows a narrow absorption with a red wing extending up to $\sim 12.0 \mathrm{~km} \mathrm{~s}^{-1}$, suggesting a blend of two independent features (Fig. 1). The $\mathrm{Ca}$ II K profile can be fit with two Gaussians centred at 4.0 and $-3.2 \mathrm{~km} \mathrm{~s}^{-1}$, and EWs $\sim 5.7$ and $\sim 4.0 \mathrm{m \AA}$, respectively (Fig. 2). While both features are far away from the expected ISM velocity in the Colorado model, the $4.0 \mathrm{~km} \mathrm{~s}^{-1}$ feature coincides with the radial velocity of the star (Table 3, and Melis et al. 2013). A Ca II $\mathrm{H}$ absorption is indistinguishable from the noise. The $\mathrm{Na}$ I doublet appears at a velocity of $5.3 \mathrm{~km} \mathrm{~s}^{-1}$, that is, at the radial velocity of the star and one of the $\mathrm{Ca}$ II $\mathrm{K}$ components (Fig. 2). Their EWs are $\sim 42 \mathrm{~m} \AA$ (Na I D2) and $\sim 34 \mathrm{~m} \AA$ (Na I D1), which suggests that the $\mathrm{Na}$ I lines are optically thick. Among the field stars around HD 131488 (see Appendix A), HD 132200 , located at a distance of $165 \mathrm{pc}$ and at an angular distance from HD 131488 of $1.24^{\circ}$, shows two non-photospheric Ca II K absorptions at velocities $-6.9 \mathrm{~km} \mathrm{~s}^{-1}$ and $4.6 \mathrm{~km} \mathrm{~s}^{-1}$, which are similar to the velocities of the observed features in HD 131488. As mentioned before, both stars belong to the complex ScorpiusCentaurus OB association, and both show a feature at negative velocities close to the mean velocity of $\sim-6.6 \mathrm{~km} \mathrm{~s}^{-1}$ that characterize the approaching face, located at a distance $\leq 76 \mathrm{pc}$, of the large expanding bubble around the OB association (Pöppel et al. 2010). Thus, the negative velocity feature of both stars is likely of ISM origin. At the same time, the $4.6 \mathrm{~km} \mathrm{~s}^{-1}$ feature of HD 132200 is variable, suggesting a relation to the star (see Fig. A.1). This fact also suggests that the HD $1314884.0 \mathrm{~km} \mathrm{~s}^{-1}$ absorption, which coincides with the stellar radial velocity, is likely circumstellar.

$H D$ 131835. This star shows a non-photospheric absorption in both $\mathrm{Ca}$ II $\mathrm{H} \& \mathrm{~K}$ lines with a weak red wing extending up to $\sim 0.0 \mathrm{~km} \mathrm{~s}^{-1}$ (Figs. 1 and 3). The feature profiles can be fit with two (Gaussian) components at $\sim-13.8 \mathrm{~km} \mathrm{~s}^{-1}$ and $\mathrm{EW} \sim 8.3 \mathrm{m \AA}$, and $-5.9 \mathrm{~km} \mathrm{~s}^{-1}$ and EW $\sim 2.1 \mathrm{m \AA}$. The corresponding Ca II 


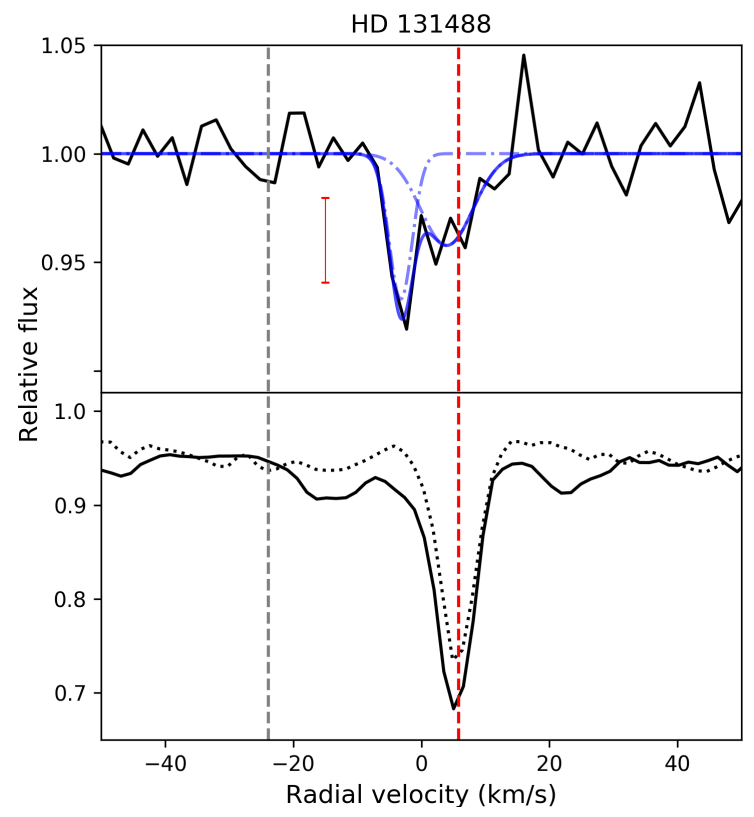

Fig. 2. Top panel: non-photospheric Ca II $\mathrm{K}$ feature profile of $\mathrm{HD}$ 131488. The observed spectrum is plotted with a black solid line. The $\mathrm{Ca}$ II $\mathrm{K}$ feature is fit with two Gaussians, plotted with a blue dash-dotted line; the blue continuous line is the sum of both Gaussians. A $3 \sigma$ error bar is plotted in red. Bottom panel: both Na I D2 (continuous line) and Na I D1 (dotted line) components.

$\mathrm{H}$ EW values are $\sim 4.1 \mathrm{~m} \AA$ and $\sim 1.4 \mathrm{~m} \AA$, respectively. A third very weak component, $\sim 3 \sigma$ level, is present only in Ca II $\mathrm{K}$ at a velocity of $2.2 \mathrm{~km} \mathrm{~s}^{-1}$ and $\mathrm{EW} \sim 0.7 \mathrm{m \AA}$ (Table 3, Figs. 1 and $3)$. This weak feature, which would require further confirmation, coincides with the radial velocity of the star. The Na I D lines show a narrow feature at $\sim-11.8 \mathrm{~km} \mathrm{~s}^{-1}$, approximately at the velocity of the strongest $\mathrm{Ca}$ II component (Fig. 3). The estimated EWs are $\sim 41 \mathrm{~m} \AA$ (Na I D2) and $\sim 32 \mathrm{~m} \AA$ (Na I D1), suggesting they are partly optically thick. We note that stars in the field show a non-photospheric absorption at $\sim-13 \mathrm{~km} \mathrm{~s}^{-1}$ (Appendix A), close to the one observed in HD 131835, and that the $-5.9 \mathrm{~km} \mathrm{~s}^{-1}$ feature is close to the mentioned mean velocity of the approaching face of the expanding bubble around the Scorpius-Centaurus association. Thus, both features are likely to be interstellar, while the weak $3 \sigma$ one has an ambiguous origin (see Appendix A).

$H D$ 138813. A clear, very broad $\left(\Delta \mathrm{v}(\mathrm{FWHM}) \sim 18.7 \mathrm{~km} \mathrm{~s}^{-1}\right)$ Ca II K absorption at $-27.5 \mathrm{~km} \mathrm{~s}^{-1}$ is detected, coinciding with the ISM velocity (Fig. 1), but it is hardly recognisable in the $\mathrm{Ca}$ II $\mathrm{H}$ line. The Na I D lines (Fig. 4) show a narrow feature at a velocity of $\sim-12 \mathrm{~km} \mathrm{~s}^{-1}$ and EWs of $18 \mathrm{~m} \AA$ (D2 line) and $15.4 \mathrm{~m} \AA$ (D1 line), suggesting that they are partly optically thick. Field stars show Na I D absorption features at similar velocities (Appendix A). Neither $\mathrm{Ca}$ II nor $\mathrm{Na}$ I features are detected at the radial velocity of the star.

$H D$ 146897. This star presents a narrow Ca II $\mathrm{K}$ absorption at the bottom of the photospheric line with an EW of $\sim 12.4 \mathrm{~m} \AA$ and a velocity of $\sim-8.0 \mathrm{~km} \mathrm{~s}^{-1}$, close to the radial velocity of the star (Fig. 1, Table 3). A second weaker feature is detectable at a velocity of $\sim-30.0 \mathrm{~km} \mathrm{~s}^{-1}$, that is, the ISM velocity vector. No absorption is recognisable in the $\mathrm{Ca}$ II $\mathrm{H}$ line. Narrow strong $\mathrm{Na}$ I $\mathrm{D}$ absorptions are detected with the same velocity as the strong Ca II K feature (Fig. 5); their EWs are $\sim 118 \mathrm{~m} \AA$ (D2 line) and $\sim 80 \mathrm{~m} \AA$ (D1 line), suggesting that they are at least partly optically thick. Field stars around HD 146897 show absorption

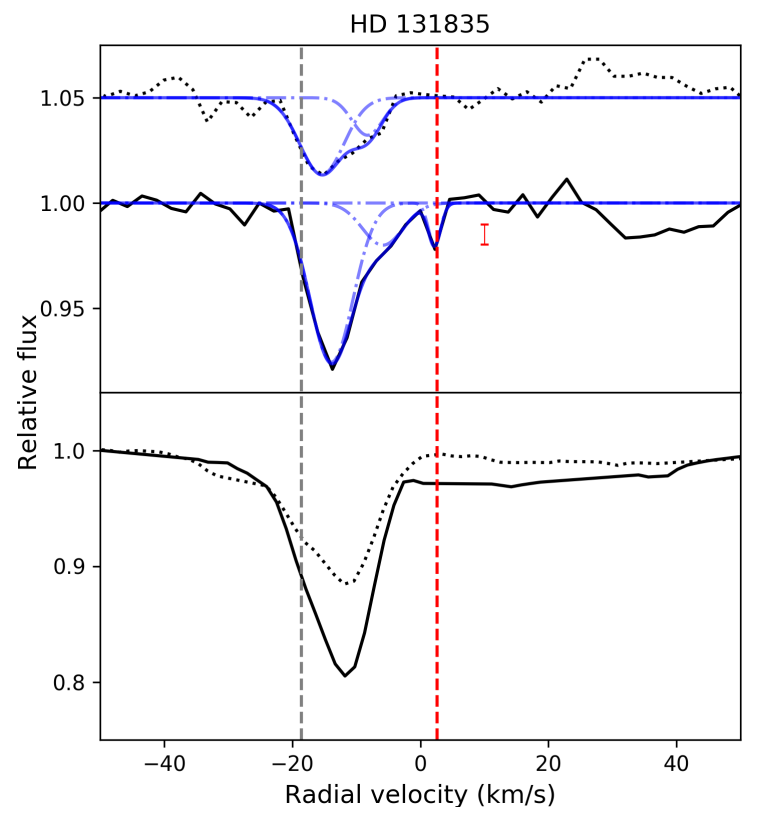

Fig. 3. Top panel: non-photospheric Ca II K (black continuous line) and $\mathrm{H}$ (black dotted lined) feature profiles of HD 131835. Ca II H has been displaced 0.05 relative units for clarity. Both $\mathrm{Ca}$ II features are fit with three and two Gaussians, respectively, plotted with blue dashdotted lines; the blue continuous lines are the sum of the Gaussians in each case. A $3 \sigma$ error bar is also plotted in red. Bottom panel: Na I D2 (black continuous line) and D1 (black dotted line) profiles.

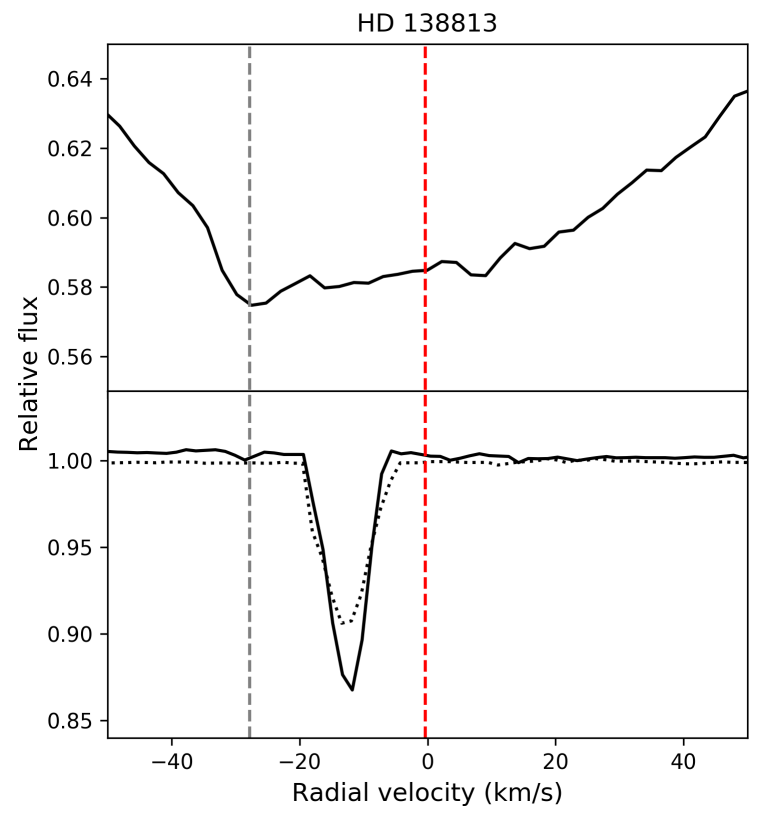

Fig. 4. Top panel: Ca II K line profile of HD 138813. An absorption feature is detected at the ISM velocity. Bottom panel: non-photospheric $\mathrm{Na}$ I D2 (black continuous line) and Na I D1 (black dotted line) absorption features.

features with similar velocities in the $\mathrm{Ca}$ II and/or $\mathrm{Na}$ I lines (Appendix A).

$H D$ 156623. Two Ca II $\mathrm{K}$ absorptions at velocities of $\sim-15.0$ and $\sim 8.1 \mathrm{~km} \mathrm{~s}^{-1}$ and with EWs of $\sim 9.0 \mathrm{~m} \AA$ and $\sim 4.3 \mathrm{~m} \AA$, respectively, are detected superimposed on the photospheric line. Neither feature exactly coincides either with the Colorado model ISM velocity vector or with the radial velocity of the star (Fig. 1 and Table 3). While the strongest, very broad feature remains 


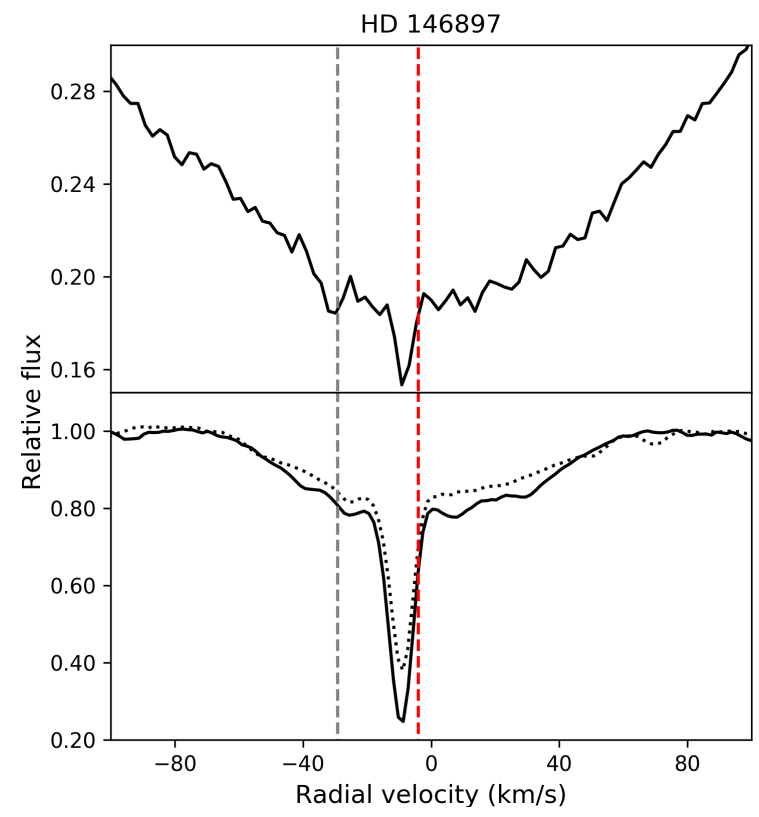

Fig. 5. Top panel: Ca II K line profile of HD 146897. Bottom panel: Na I D2 (black continuous line) and Na I D1 (black dotted line).

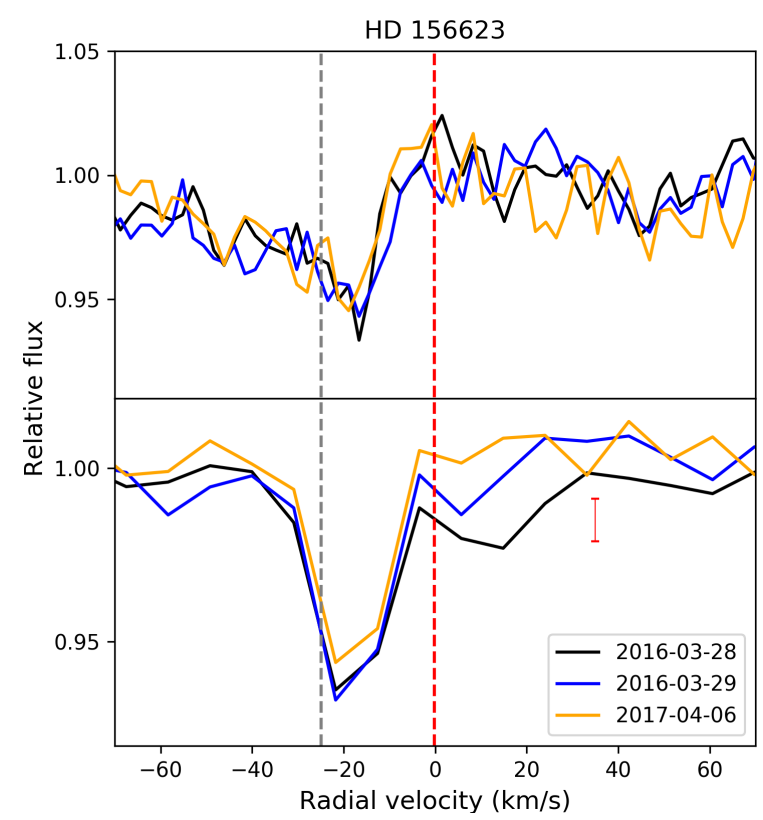

Fig. 6. Top panel: individual non-photospheric $\mathrm{Ca}$ II $\mathrm{H}$ absorption features of HD 156623. Bottom panel: Ca II K line. The variable redshifted absorption at $\sim 8.1 \mathrm{~km} \mathrm{~s}^{-1}$ is a clear FEB-like event. A $3 \sigma$ error bar is plotted in red. Dates are indicated with different colours in both panels.

practically constant, the weaker one shows variability. As an example, Fig. 6 shows three Ca II K spectra of HD 156623 taken at different epochs where it is distinguished that the $\sim 8.1 \mathrm{~km} \mathrm{~s}^{-1}$ absorption is clearly a FEB-like event. A Ca II $\mathrm{H}$ feature at the velocity of the strongest $\mathrm{Ca}$ II $\mathrm{K}$ with $\mathrm{EW} \sim 3.7 \mathrm{~m} \AA$ is present in our spectra. Neither CS or ISM absorptions are detected in the $\mathrm{Na}$ I $\mathrm{D}$ lines.

$H D$ 172555. A narrow $\mathrm{Ca}$ II $\mathrm{K}$ absorption with a velocity of $2.3 \mathrm{~km} \mathrm{~s}^{-1}$ and an EW of $\sim 9.9 \mathrm{m \AA}$ at the core of the stellar line is detected (Fig. 1 and Table 3). A similar absorption is detected in the $\mathrm{Ca}$ II H line with an EW( $\mathrm{Ca}$ II H) of $\sim 4.0 \mathrm{~m} \AA$. No feature is detected in the $\mathrm{Na}$ I D lines. Similar $\mathrm{Ca}$ II and $\mathrm{Na}$ I results were

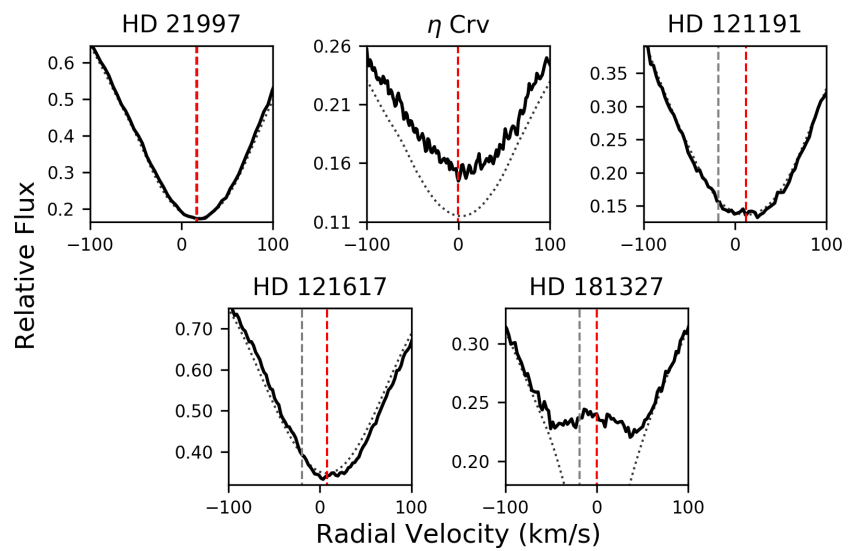

Fig. 7. Observed median Ca II $\mathrm{K}$ line profiles of cold-gas-bearing debris-disc stars without non-photospheric absorption features.

found by Kiefer et al. (2014a), although those authors detected some Ca II FEBs not present in any of our spectra.

HD $181296(\eta \mathrm{Tel})$. A narrow absorption is observed in both $\mathrm{Ca}$ II $\mathrm{H} \& \mathrm{~K}$ lines at a velocity of $\sim-22.8 \mathrm{~km} \mathrm{~s}^{-1}$ with EWs of $\sim 3.9 \mathrm{~m} \AA$ (K-line) and $\sim 2.2 \mathrm{m \AA}$ (H-line). No feature is detected in the $\mathrm{Na}$ I D lines. The Ca II feature velocity is very different from the estimated radial velocity of the star of $-3 \mathrm{~km} \mathrm{~s}^{-1}$. The line of sight to this star does not traverse any identified local cloud in the Colorado model, but it is close $\left(<20^{\circ}\right)$ to some of them; in particular to the $\mathrm{G}$ and Vel clouds which have a projected velocity of -18.51 and $-27.6 \mathrm{~km} \mathrm{~s}^{-1}$, respectively.

\subsection{Stars without narrow absorption components}

Neither stable nor variable $\mathrm{Ca}$ II or Na I absorption features, which could be attributed to the ISM or CS medium, have been detected in any of our spectra towards the cold-gas-bearing debris-disc stars HD 21997, $\eta$ Crv, HD 121191, HD 121617, and HD 181327. For the sake of completeness, Fig. 7 shows the observed median Ca II K profile of these stars together with the Kurucz synthetic profiles. A clear emission due to stellar activity at the core of the photospheric line is apparent in the spectrum of the F6 V star HD 181327. At the same time, the observed Ca II $\mathrm{K}$ line of $\eta \mathrm{Crv}$ (HD 109085, F2 V spectral type) is not as deep as predicted by the synthetic line. We have tested the effects of changing the metallicity and $\mathrm{Ca}$ abundance in the Kurucz models without finding a satisfactory fit between the observed Ca II K line and the synthetic one. However, we have achieved satisfactory fits between Kurucz models and stars with similar properties as $\eta \mathrm{Crv}$. These results lead us to conclude that $\eta \mathrm{Crv}$ most likely presents some level of chromospheric activity.

\section{Discussion}

Before discussing plausible origins of the observed nonphotospheric features and their potential correlations with the properties of the cold-gas-bearing debris discs and their host stars, it is useful to globally evaluate the observational results of the whole cold-gas-bearing debris-disc sample, that is, including the two systems not observed by us, $\beta$ Pic and Fomalhaut.

\subsection{Global overview of the observational results}

As pointed out before, 10 out of the 15 observed gas-bearing debris-disc stars show at least one non-photospheric Ca II absorption feature. Table 4 summarizes these observational 
I. Rebollido et al.: The co-existence of hot and cold gas in debris discs

Table 4. Summary of the non-photospheric absorption features of the observed cold-gas-bearing debris-disc stars.

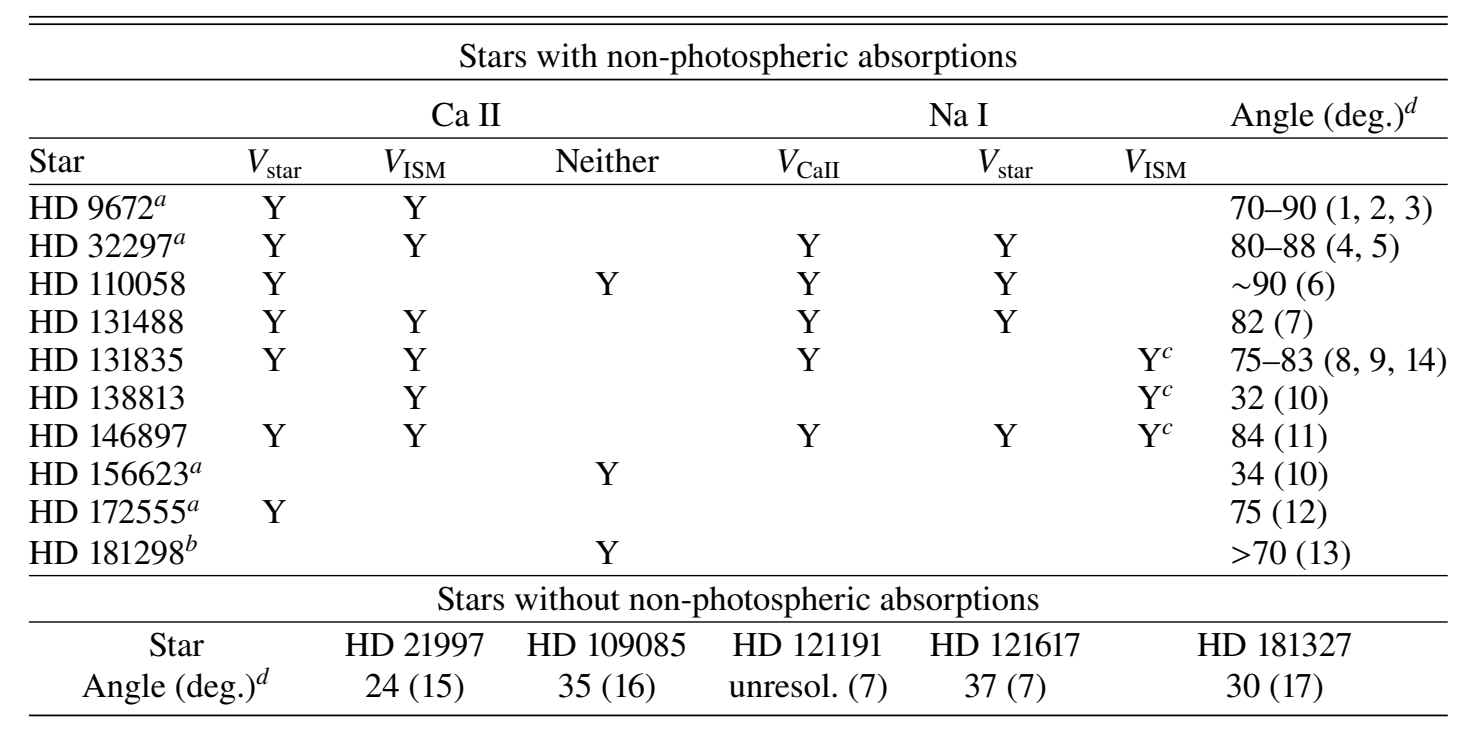

Notes. Columns 2 to 4: coincidences of the Ca II velocity components with the radial velocity of the star, Colorado model ISM clouds along the line of sight, or none of both. Columns 5 to 7: coincidences of the Na I velocity components with Ca II features, the radial velocity of the star, or ISM absorptions. Column 8 gives the inclination angle of the debris disc associated with the stars. Empty spaces signify non-detections. ${ }^{(a)}$ Stars with FEBs. ${ }^{(b)}$ See text Sec. 5.2. ${ }^{(c)}$ ISM absorptions from field stars. ${ }^{(d)}$ References for inclination angle: (1) Hughes et al. (2008); (2) Moór et al. (2015b); (3) Choquet et al. (2017); (4) Schneider et al. (2005); (5) Asensio-Torres et al. (2016); (6) Kasper et al. (2015); (7) Moór et al. (2017); (8) Hung et al. (2015); (9) Moór et al. (2015a); (10) Hales et al. (in prep.); (11) Thalmann et al. (2013); (12) Smith et al. (2012); (13) Smith et al. (2009); (14) Feldt et al. (2017);(15) Moór et al. (2013); (16) Marino et al. (2017); (17) Marino et al. (2016) .

findings, where we indicate the stars with non-photospheric Ca II and $\mathrm{Na}$ I features at the velocities of the star, ISM clouds of the Colorado model, and/or plausible ISM absorptions as suggested by their detection in field stars. We have conservatively assumed that the observed features have a stellar or ISM counterpart if the velocity difference is less than $5 \mathrm{~km} \mathrm{~s}^{-1}$. The table also shows those stars with FEB-like events.

When considering the whole sample of 17 cold-gas-bearing debris-disc stars, we find that 11 of them (including $\beta$ Pic) show at least one non-photospheric $\mathrm{Ca}$ II $\mathrm{K}$ absorption. That leaves 6 objects with no traces of any extra absorption superimposed on the photospheric line. Fomalhaut has been added to the observed stars in this work since neither $\mathrm{Ca}$ II nor $\mathrm{Na}$ I extra absorptions in the core of the stellar lines have been detected (Hobbs 1986; Lagrange-Henri et al. 1990; Holweger \& Rentzsch-Holm 1995). Furthermore, we have downloaded UVES spectra from the ESO archive confirming the lack of a non-photospheric absorption feature in the Fomalhaut spectrum.

\subsection{Origin of the non-photospheric absorption features}

There is some ambiguity concerning the CS or ISM origin of the stable $\mathrm{Ca}$ II and $\mathrm{Na}$ I absorptions as they can originate in warm and cold clouds of the local ISM. The observed line EWs, their ratios, and (in most cases) the velocity dispersion of the $\mathrm{Ca}$ II $\mathrm{K}$ line are not different from what is detected in the ISM (Redfield \& Linsky 2002; Welsh et al. 2010), where different lines of sight with or without $\mathrm{Ca}$ II or $\mathrm{Na}$ I absorptions are found, as well as a large range of $\mathrm{Ca}$ II-to-Na I ratios (Redfield et al. 2007; Welsh et al. 2010). Nonetheless, a CS origin has been attributed to at least one velocity component in the cases of 49 Ceti, HD 32297, HD 110058, and HD 172555 (e.g. Montgomery \& Welsh 2012; Redfield et al. 2007; Hales et al. 2017; Kiefer et al. 2014a), respectively. HD 156623 shows a variable FEB-like event. The feature at the stellar radial velocity of
HD 131488 is likely CS (Sect. 4.1.1). The weak $3 \sigma$ feature, if confirmed, at the radial velocity of HD 131835 might also be circumstellar. At the same time, an ISM origin is the most plausible case for the non-photospheric absorptions in HD 138813 and HD 146897, although even in these two stars the Ca II K FWHM dispersion velocities, $\sim 18.7 \mathrm{~km} \mathrm{~s}^{-1}$, are larger than typical ISM values (e.g. Redfield \& Linsky 2002).

HD 181296 ( $~($ Tel) merits particular attention. This star has a stable feature blueshifted by $\sim 20 \mathrm{~km} \mathrm{~s}^{-1}$ with respect to the stellar radial velocity (Fig. 1 and Table 3); its line of sight does not cross any of the Colorado model clouds, although it passes within $20^{\circ}$ of several clouds. $\eta$ Tel is located at a projected angular distance of $\sim 7$ arcmin from HD 181327, which corresponds to $\sim 0.1 \mathrm{pc}$ at the distance of both stars (Table 2). HD 181327 is another cold-gas debris disc but without non-photospheric absorption features (Table 3 and Fig. 7). Both stars are members of the $\beta$ Pic moving group, and form part of a triple system (Neuhäuser et al. 2011), sharing distances, radial velocities, and proper motions. Thus, although an ISM origin cannot be totally excluded from being the $\eta$ Tel Ca II absorption feature, those data strongly suggest that the feature is CS. We note that similar blueshifted CS absorptions in UV-excited lines of C II and N II are found around the debris-disc star $\sigma$ Her, which has a similar $T_{\text {eff }}$ as $\eta$ Tel (Chen \& Jura 2003). Those authors suggest that the blueshifted features arise in a radiatively driven wind, and that the gas could be generated by the sublimation of comets or collisions between solid bodies.

The previous arguments strongly suggest the presence of hot $\mathrm{Ca}$ II and $\mathrm{Na}$ I gas in the CS environment of at least eight (including $\beta$ Pic), or possibly nine (if the feature at the stellar radial velocity of HD 131835 is confirmed) of the cold-gas-bearing debris-disc stars. Furthermore, the presence/absence of Ca II and $\mathrm{Na}$ I suggests that there are differences in the CS gas composition around the debris-disc stars. We note that Kiefer et al. (2014b) 
suggest the presence of two families of exocomets in $\beta$ Pic, one of them strongly depleted in volatiles. Assuming, therefore, the plausible circumstellar-hot-gas scenario as suggested by some of the non-photospheric features, the permanence of such gas in the surroundings of the stars would require a continuous replenishment as it would be subject to a strong radiation field overcoming the gravitational force (Beust et al. 1989). These authors, and Lagrange et al. (1998), Liseau (2003), and Fernández et al. (2006) have estimated the ratio $\beta=F_{\text {rad }} / F_{\text {grav }}$ in the case of $\beta$ Pic for lines of many different atoms/species, including the $\mathrm{Ca}$ II H\&K and $\mathrm{Na}$ I lines, and shown that the atomic gas would disappear unless a braking mechanism is at work. This would also be the scenario of the stars in this work as their radiation fields are similar to that of $\beta$ Pic.

Thus, a braking mechanism should be at work to explain the hot, inner $(<1 \mathrm{au}) \mathrm{CS}$ gas around these gas-bearing debrisdisc stars. Such a mechanism could be the one suggested by Fernández et al. (2006)-acceleration of an ionic fluid produced by an enhanced carbon abundance. In this sense, it would be interesting to search for carbon lines around these stars, for example in the UV spectral range (Roberge et al. 2006, 2014).

\subsection{Correlation of the non-photospheric features with the inclination of the debris discs}

We have checked if any observational property of the stars or of the gas/dust components in their debris discs correlate in some way with the presence/absence and strength of the $\mathrm{Ca}$ II/Na I absorptions at the core of the stellar lines. We note that CS features are only detected in the A-type stars of the sample and in none of the three F-type stars, but we cannot identify any trend with other properties like rotational velocity or age.

Concerning the debris disc properties, the only trend we find is that non-photospheric CS absorptions are predominantly detected when the discs are oriented close to edge-on. This is the case in at least 7 (possibly 8 if HD 131835 is included) out of 9 objects. On the contrary, discs seen close to face-on ( 8 objects) do not show non-photospheric absorptions, with the exception of HD 156623 that shows evidence of hot gas as suggested by the FEB-like absorption (Table 4 ). $\beta$ Pic and Fomalhaut are included in those figures, as the $\beta$ Pic disc is seen edge-on, $\sim 90^{\circ}$ (Smith \& Terrile 1984), but not the Fomalhaut disc, $\sim 65^{\circ}$ (Acke et al. 2012). Thus, at least $\sim 78 \%$ (possibly $88 \%$ ) of edgeon cold-gas-bearing debris-disc stars show evidence of hot CS gas, while only $\sim 12 \%$ of the face-on cold-gas-bearing debrisdisc stars show such evidence. While this result is not surprising as such, it suggests that the non-detection is most likely due to geometrical effects, that is, the disc inclination with respect to the line of sight, but not to the absence of hot gas in those discs. In the case of HD 156623, the detection of the FEB-like Ca II event, in addition to the very broad stable absorption (Table 3 ), might indicate a significant scale height of the hot gas, since such gas would be located very close to the star. Based on the $\mathrm{CO}$ gas detection in $\beta$ Pic, Kral et al. $(2016,2017)$ have recently developed a model predicting the detection with ALMA of cold gas around debris-disc stars assuming that the gas arises from icy, volatile-rich planetesimals.

From the observational perspective presented in this work, our results strongly suggest that debris discs with central absorptions indicative of hot CS gas would be good targets to search for a cold-gas counterpart with ALMA.

With respect to other debris-disc properties, all but HD 121191 have been resolved either in their dust thermal emission, gas emission, and/or scattered light (e.g.
Asensio-Torres et al. 2016; Schneider et al. 2005, 2014; Feldt et al. 2017; Choquet et al. 2017; Moór et al. 2017, see also the Catalogue of Resolved Debris Disc ${ }^{3}$ and references therein). Concerning the cold gas, neither the detected species nor the flux/luminosity of the lines show any trend with the optical absorption lines-see e.g. Moór et al. (2017) for CO line luminosities. With regard to the dust, all systems but HD 172555 present two-temperature SEDs. Kennedy \& Wyatt (2014), and Geiler \& Krivov (2017) have analysed large samples of twotemperature debris-disc systems, and have shown that most of them likely have a two-belt disc structure, similar to the solar system's zodiacal and Kuiper belts. There are, however, a small number of exceptions. In particular, HD 181327 seems to be surrounded by an extended disc with a range of dust temperatures and properties (Lebreton et al. 2012; Kennedy \& Wyatt 2014; Ballering et al. 2017). Several works postulate an exo-asteroid belt and/or cometary sources as the possible origin of the warm disc (Kennedy \& Wyatt 2014; Ballering et al. 2017; Geiler \& Krivov 2017). In any case, although both dust belts and hot gas likely share a similar, comet-related origin, we cannot identify any trend of the dust-disc properties - fractional luminosities, radii, and temperatures-with the presence or strength of the hotgas absorptions. It is interesting to note that the prototype FEB host $\beta$ Pic also harbours species of very hot dust in addition to hot gas (Defrère et al. 2012). HD 172555 also harbors both hot dust and gas (Ertel et al. 2014, 2016). The origin of this hot dust is still unclear. Although the samples of stars checked for hot dust and those for hot gas have little overlap so far, and thus a correlation cannot be seen, it seems conceivable that both phenomena might be related.

\section{Conclusions}

In this work we present high-resolution optical spectra of the currently known cold-gas-bearing debris-disc systems. The observations show $\mathrm{Ca}$ II and $\mathrm{Na}$ I non-photospheric absorptions in at least $\sim 78 \%$, and possibly $88 \%$, of the discs seen edge-on, and in only $\sim 12 \%$ of the face-on discs. Those absorptions are usually interpreted as signposts of hot gas released by exocomets or small grains in the close vicinity of the central stars. Thus, these detection rates suggest that the non-detections in some discs seen close to face-on are most likely due to geometrical effects, that is, the inclination of the CS disc. At the same time, hot gas is only detected in A-type debris-disc systems, but it does not correlate either with any other stellar or disc properties.

The optical, infrared and submillimetre observations suggest that planetesimals/cometary bodies often populate gas-bearing debris-disc systems, from a few stellar radii up to hundreds of au. Collisions among those large bodies, as well as among small dust grains, and their evaporation are the agents, possibly accompanied by planets, as in the case of $\beta$ Pic, structuring the CS environments and planetary systems of main-sequence stars. Choquet et al. (2017) have recently outlined a scenario to interpret the gas and dust structure of the 49 Ceti system; the authors suggest physical mechanisms for the production of gas at different distances from the central star. That scenario is likely at least roughly applicable to the systems studied in this work.

Acknowledgements. Based on observations made with the Mercator Telescope, operated on the island of La Palma by the Flemmish Community, and the Nordic Optical Telescope, operated by the Nordic Optical Telescope Scientific Association, at the Spanish Observatorio del Roque de los Muchachos of the Instituto

3 http://www .astro.uni-jena.de/index.php/theory/ catalog-of-resolved-debris-disks.html 
I. Rebollido et al.: The co-existence of hot and cold gas in debris discs

de Astrofísica de Canarias. Based on observations made with ESO Telescopes at the La Silla Observatory under programmes 099.A-9004(A) and 099.A-9029(A). Also based on observations made with the TIGRE telescope funded and operated by the universities of Hamburg, Guanajuato and Liège. Partially based on data obtained from the ESO Science Archive Facility. Paula Sarkis kindly provided observations of HD 121191. We thank A. Hales for sharing with us the disc inclination of HD 138813 and HD 156623 before publication. H.C., C.E., A.G. G.M., B.M., I.R., and E.V. are supported by Spanish grant AYA 2014-55840P. H.C. acknowledges funding from the ESA Research Fellowship Programme. D.P.I. thanks Iván Lacerna for his help during the observations in La Silla in October 2015. This work has made use of data from the European Space Agency (ESA) mission Gaia (https://www.cosmos.esa.int/gaia), processed by the Gaia Data Processing and Analysis Consortium (DPAC, https://www. cosmos.esa.int/web/gaia/dpac/consortium). Funding for the DPAC has been provided by national institutions, in particular the institutions participating in the Gaia Multilateral Agreement. The work of A.M. was supported by the Momentum grant of the MTA CSFK Lenduilet Disk Research Group and Hungarian OTKA grant K125015. We thank the anonymous referee for his/her comments and suggestions, which helped to improve the paper.

\section{References}

Acke, B., Min, M., Dominik, C., et al. 2012, A\&A, 540, A125

Asensio-Torres, R., Janson, M., Hashimoto, J., et al. 2016, A\&A, 593, A73

Backman, D. E., \& Paresce, F. 1993, in Protostars and Planets III, eds. E. H. Levy \& J. I. Lunine, 1253

Ballering, N. P., Rieke, G. H., Su, K. Y. L., \& Montiel, E. 2013, ApJ, 775, 55

Ballering, N. P., Rieke, G. H., Su, K. Y. L., \& Gáspár, A. 2017, ApJ, 845, 120

Bell, C. P. M., Mamajek, E. E., \& Naylor, T. 2015, MNRAS, 454, 593

Beust, H., \& Morbidelli, A. 2000, Icarus, 143, 170

Beust, H., Lagrange-Henri, A. M., Vidal-Madjar, A., \& Ferlet, R. 1989, A\&A, 223, 304

Beust, H., Vidal-Madjar, A., Ferlet, R., \& Lagrange-Henri, A. M. 1990, A\&A, 236, 202

Beust, H., Vidal-Madjar, A., \& Ferlet, R. 1991, A\&A, 247, 505

Beust, H., Lagrange, A.-M., Crawford, I. A., et al. 1998, A\&A, 338, 1015

Brandeker, A. 2011, ApJ, 729, 122

Brandeker, A., Liseau, R., Olofsson, G., \& Fridlund, M. 2004, A\&A, 413, 681

Castelli, F., \& Kurucz, R. L. 2004, ArXiv e-prints [arXiv: astro-ph/0405087]

Cataldi, G., Brandeker, A., Olofsson, G., et al. 2014, A\&A, 563, A66

Chen, C. H., \& Jura, M. 2003, ApJ, 582, 443

Chen, C. H., Mittal, T., Kuchner, M., et al. 2014, ApJS, 211, 25

Choquet, É., Milli, J., Wahhaj, Z., et al. 2017, ApJ, 834, L12

Cotten, T. H., \& Song, I. 2016, ApJS, 225, 15

de Bruijne, J. H. J., \& Eilers, A.-C. 2012, A\&A, 546, A61

Defrère, D., Lebreton, J., Le Bouquin, J.-B., et al. 2012, A\&A, 546, L9

Dent, W. R. F., Wyatt, M. C., Roberge, A., et al. 2014, Science, 343, 1490

Donaldson, J. K., Lebreton, J., Roberge, A., Augereau, J.-C., \& Krivov, A. V. 2013, ApJ, 772, 17

Eiroa, C., Rebollido, I., Montesinos, B., et al. 2016, A\&A, 594, L1

Ertel, S., Absil, O., Defrère, D., et al. 2014, A\&A, 570, A128

Ertel, S., Defrère, D., Absil, O , et al. 2016, A\&A, 595, A44

Feldt, M., Olofsson, J., Boccaletti, A., et al. 2017, A\&A, 601, A7

Ferlet, R., Vidal-Madjar, A., \& Hobbs, L. M. 1987, A\&A, 185, 267

Fernández, R., Brandeker, A., \& Wu, Y. 2006, ApJ, 643, 509

Gebran, M., Farah, W., Paletou, F., Monier, R., \& Watson, V. 2016, A\&A, 589, A83

Geiler, F., \& Krivov, A. V. 2017, MNRAS, 468, 959

Greaves, J. S., Holland, W. S., Matthews, B. C., et al. 2016, MNRAS, 461, 3910

Hales, A. S., Barlow, M. J., Crawford, I. A., \& Casassus, S. 2017, MNRAS, 466 3582

Hobbs, L. M. 1986, ApJ, 308, 854

Hobbs, L. M., Vidal-Madjar, A., Ferlet, R., Albert, C. E., \& Gry, C. 1985, ApJ, 293, L29

Hobbs, L. M., Welty, D. E., Lagrange-Henri, A. M., Ferlet, R., \& Vidal-Madjar, A. 1988, ApJ, 334, L41

Holweger, H., \& Rentzsch-Holm, I. 1995, A\&A, 303, 819

Hughes, A. M., Wilner, D. J., Kamp, I., \& Hogerheijde, M. R. 2008, ApJ, 681 , 626

Hung, L.-W., Fitzgerald, M. P., Chen, C. H., et al. 2015, ApJ, 802, 138

Kalas, P. 2005, ApJ, 635, L169

Kasper, M., Apai, D., Wagner, K., \& Robberto, M. 2015, ApJ, 812, L33

Kaufer, A., Stahl, O., Tubbesing, S., et al. 1999, The Messenger, 95, 8

Kennedy, G. M., \& Wyatt, M. C. 2014, MNRAS, 444, 3164

Kiefer, F., Lecavelier des Etangs, A., Augereau, J.-C., et al. 2014a, A\&A, 561, L10
Kiefer, F., Lecavelier des Etangs, A., Boissier, J., et al. 2014b, Nature, 514, 462 Kondo, Y., \& Bruhweiler, F. C. 1985, ApJ, 291, L1

Kóspál, Á., Moór, A., Juhász, A., et al. 2013, ApJ, 776, 77

Kral, Q., Wyatt, M., Carswell, R. F., et al. 2016, MNRAS, 461, 845

Kral, Q., Matrà, L., Wyatt, M. C., \& Kennedy, G. M. 2017, MNRAS, 469, 521

Kurucz, R. L. 1993, Peculiar versus Normal Phenomena in A-type and Related Stars, IAU Colloq. 138, ASP Conf. Ser., 44, 87

Lagrange, A.-M., Beust, H., Mouillet, D., et al. 1998, A\&A, 330, 1091

Lagrange-Henri, A. M., Vidal-Madjar, A., \& Ferlet, R. 1988, A\&A, 190, 275

Lagrange-Henri, A. M., Ferlet, R., Vidal-Madjar, A., et al. 1990, A\&AS, 85, 1089

Lebreton, J., Augereau, J.-C., Thi, W.-F., et al. 2012, A\&A, 539, A17

Lieman-Sifry, J., Hughes, A. M., Carpenter, J. M., et al. 2016, ApJ, 828, 25

Lindegren, L., Lammers, U., Bastian, U., et al. 2016, A\&A, 595, A4

Liseau, R. 2003, in Earths: DARWIN/TPF and the Search for Extrasolar Terrestrial Planets, eds. M. Fridlund, T. Henning, \& H. Lacoste, ESA SP, 539, 135

Malamut, C., Redfield, S., Linsky, J. L., Wood, B. E., \& Ayres, T. R. 2014, ApJ 787, 75

Mamajek, E. E. 2012, ApJ, 754, L20

Mamajek, E. E., \& Bell, C. P. M. 2014, MNRAS, 445, 2169

Marino, S., Matrà, L., Stark, C., et al. 2016, MNRAS, 460, 2933

Marino, S., Wyatt, M. C., Panić, O., et al. 2017, MNRAS, 465, 2595

Matrà, L., Dent, W. R. F., Wyatt, M. C., et al. 2017, MNRAS, 464, 1415

Matthews, B. C., Krivov, A. V., Wyatt, M. C., Bryden, G., \& Eiroa, C. 2014, Protostars and Planets VI, eds. H., Beuther, R. S., Klessen, C. P., Dullemond, $\&$ T., Henning (Tucson: Univ. of Arizona Press), 521

Melis, C., Zuckerman, B., Rhee, J. H., et al. 2013, ApJ, 778, 12

Montgomery, S. L., \& Welsh, B. Y. 2012, PASP, 124, 1042

Moór, A., Ábrahám, P., Juhász, A., et al. 2011, ApJ, 740, L7

Moór, A., Juhász, A., Kóspál, Á., et al. 2013, ApJ, 777, L25

Moór, A., Henning, T., Juhász, A., et al. 2015a, ApJ, 814, 42

Moór, A., Kóspál, Á., Ábrahám, P., et al. 2015b, MNRAS, 447, 577

Moór, A., Curé, M., Kóspál, Á., et al. 2017, ApJ, 849, 123

Neuhäuser, R., Ginski, C., Schmidt, T. O. B., \& Mugrauer, M. 2011, MNRAS, 416,1430

Olofsson, G., Liseau, R., \& Brandeker, A. 2001, ApJ, 563, L77

Pecaut, M. J., \& Mamajek, E. E. 2016, MNRAS, 461, 794

Pecaut, M. J., Mamajek, E. E., \& Bubar, E. J. 2012, ApJ, 746, 154

Pöppel, W. G. L., Bajaja, E., Arnal, E. M., \& Morras, R. 2010, A\&A, 512, A83

Rappaport, S., Vanderburg, A., Jacobs, T., et al. 2018, MNRAS, 474, 1453

Raskin, G., van Winckel, H., Hensberge, H., et al. 2011, A\&A, 526, A69

Redfield, S., \& Linsky, J. L. 2002, ApJS, 139, 439

Redfield, S., \& Linsky, J. L. 2008, ApJ, 673, 283

Redfield, S., Kessler-Silacci, J. E., \& Cieza, L. A. 2007, ApJ, 661, 944

Riviere-Marichalar, P., Barrado, D., Augereau, J.-C., et al. 2012, A\&A, 546, L8

Riviere-Marichalar, P., Barrado, D., Montesinos, B., et al. 2014, A\&A, 565, A68

Roberge, A., \& Weinberger, A. J. 2008, ApJ, 676, 509

Roberge, A., Feldman, P. D., Lagrange, A. M., et al. 2000, ApJ, 538, 904

Roberge, A., Feldman, P. D., Weinberger, A. J., Deleuil, M., \& Bouret, J.-C. 2006, Nature, 441, 724

Roberge, A., Kamp, I., Montesinos, B., et al. 2013, ApJ, 771, 69

Roberge, A., Welsh, B. Y., Kamp, I., Weinberger, A. J., \& Grady, C. A. 2014 ApJ, 796, L11

Schmitt, J. H. M. M., Schröder, K.-P., Rauw, G., et al. 2014, Astron. Nachr., 335, 787

Schneider, G., Silverstone, M. D., \& Hines, D. C. 2005, ApJ, 629, L117

Schneider, G., Grady, C. A., Hines, D. C., et al. 2014, AJ, 148, 59

Slettebak, A. 1975, ApJ, 197, 137

Smith, B. A., \& Terrile, R. J. 1984, Science, 226, 1421

Smith, R., Churcher, L. J., Wyatt, M. C., Moerchen, M. M., \& Telesco, C. M. 2009, A\&A, 493, 299

Smith, R., Wyatt, M. C., \& Haniff, C. A. 2012, MNRAS, 422, 2560

Telting, J. H., Avila, G., Buchhave, L., et al. 2014, Astron. Nachr., 335, 41

Thalmann, C., Janson, M., Buenzli, E., et al. 2013, ApJ, 763, L29

Thureau, N. D., Greaves, J. S., Matthews, B. C., et al. 2014, MNRAS, 445, 2558

Torres, C. A. O., Quast, G. R., Melo, C. H. F., \& Sterzik, M. F. 2008, Young

Nearby Loose Associations, ed. B. Reipurth, 757

van Dishoeck, E. F., \& Black, J. H. 1988, ApJ, 334, 771

van Leeuwen F. 2007, A\&A, 474, 653

Vidal-Madjar, A., Lagrange-Henri, A.-M., Feldman, P. D., et al. 1994, A\&A, 290,245

Vidal-Madjar, A., Kiefer, F., Lecavelier des Etangs, A., et al. 2017, A\&A, 607, A25

Welsh, B. Y., \& Montgomery, S. L. 2015, Adv. Astron., 2015, 980323

Welsh, B. Y., \& Montgomery, S. L. 2018, MNRAS, 474, 1515

Welsh, B. Y., Lallement, R., Vergely, J.-L., \& Raimond, S. 2010, A\&A, 510, A54

Wenger, M., Ochsenbein, F., Egret, D., et al. 2000, A\&AS, 143, 9

Wyatt, M. C. 2008, ARA\&A, 46, 339

Zuckerman, B., Forveille, T., \& Kastner, J. H. 1995, Nature, 373, 494 
Appendix A: Field stars

Table A.1. HD 131488: field stars.

\begin{tabular}{lrrr}
\hline \hline Star & $\begin{array}{r}\mathrm{r} \\
(\mathrm{deg} .)\end{array}$ & $\begin{array}{r}\mathrm{v}_{\mathrm{rad}} \\
\left(\mathrm{km} \mathrm{s}^{-1}\right)\end{array}$ & $\begin{array}{r}\mathrm{v}_{\text {CaIIK }} \\
\left(\mathrm{km} \mathrm{s}^{-1}\right)\end{array}$ \\
\hline HD 131488 & & 5.8 & $-3.2 / 4.0$ \\
\hline HD 128207 & 3.60 & 2.0 & -13.0 \\
HD 131120 & 3.35 & 6.1 & -16.0 \\
HD 132058 & 2.11 & 0.2 & -19.1 \\
HD 132094 & 3.81 & 0.9 & -11.4 \\
HD 132200 & 1.24 & 8.0 & $-6.9 / 4.6$ \\
HD 133880 & 2.53 & 2.8 & -13.0 \\
HD 133937 & 3.06 & 2.0 & -21.4 \\
HD 135454 & 4.20 & 1.4 & -24.4 \\
\hline
\end{tabular}

Table A.2. HD 138813: field stars.

\begin{tabular}{lrrr}
\hline \hline Star & $\begin{array}{r}\mathrm{r} \\
(\text { deg. })\end{array}$ & $\begin{array}{r}\mathrm{v}_{\text {rad }} \\
\left(\mathrm{km} \mathrm{s}^{-1}\right)\end{array}$ & $\begin{array}{r}\mathrm{v}_{\text {NaID2 }} \\
\left(\mathrm{km} \mathrm{s}^{-1}\right)\end{array}$ \\
\hline HD 138813 & & -0.4 & -12.0 \\
\hline HD 141637 & 3.54 & -3.0 & $-13.3 /-8.7$ \\
HD 136246 & 4.16 & -2.7 & -16.0 \\
HD 142301 & 4.40 & -8.7 & -14.3 \\
HD 142184 & 4.58 & -9.2 & -13.3 \\
HD 142250 & 4.59 & -1.3 & -12.8 \\
\hline
\end{tabular}

Table A.3. HD 131835: field stars.

\begin{tabular}{lrrr}
\hline \hline Star & $\begin{array}{r}\mathrm{r} \\
(\mathrm{deg} .)\end{array}$ & $\begin{array}{r}\mathrm{v}_{\text {rad }} \\
\left(\mathrm{km} \mathrm{s}^{-1}\right)\end{array}$ & $\begin{array}{r}\mathrm{v}_{\text {CaIIK }} \\
\left(\mathrm{km} \mathrm{s}^{-1}\right)\end{array}$ \\
\hline HD 131835 & & 2.6 & $-13.8 /-5.9 / 2.2$ \\
\hline HD 131120 & 2.26 & 6.1 & -16.0 \\
HD 132094 & 1.69 & 0.9 & -11.4 \\
HD 132955 & 3.30 & 9.5 & $-13.7 / 3.8$ \\
\hline
\end{tabular}

Table A.4. HD 146897: field stars.

\begin{tabular}{lrrrr}
\hline \hline Star & $\begin{array}{r}\mathrm{r} \\
(\mathrm{deg} .)\end{array}$ & $\begin{array}{r}\mathrm{v}_{\text {rad }} \\
\left(\mathrm{km} \mathrm{s}^{-1}\right)\end{array}$ & $\begin{array}{r}\mathrm{v}_{\text {CaIIK }} \\
\left(\mathrm{km} \mathrm{s}^{-1}\right)\end{array}$ & $\begin{array}{r}\mathrm{v}_{\text {NaID2 }} \\
\left(\mathrm{km} \mathrm{s}^{-1}\right)\end{array}$ \\
\hline HD 146897 & & -4.0 & $-30.0,-8.0$ & -8.8 \\
\hline HD 144587 & 3.76 & 0.0 & - & $-22.5,-9.2$ \\
HD 145554 & 2.47 & -6.1 & $-25.9,-9.2$ & $-25.5,-10.2$ \\
HD 145631 & 2.47 & -5.6 & $-27.5,-9.2$ & $-25.5,-10.2$ \\
HD 145964 & 1.20 & -7.8 & $-25.2,-9.2$ & $-22.5,-9.2$ \\
HD 147137 & 1.23 & -0.8 & -9.2 & -8.7 \\
HD 147932 & 2.45 & & -8.4 & -9.5 \\
\hline
\end{tabular}

This section provides information on non-photospheric absorption features of field stars around HD 131488, HD 131835, HD 138813, and HD 146897. These four stars were selected due to the ambiguous origin of their non-photospheric absorptions. The data are based on a search of stars in a field of $5^{\circ}$ in radius around each of the four stars, and parallaxes between 6 and 10 mas, so that the field stars are located at comparable distances of the cold-gas-bearing debris-disc stars. We recall that they are members of the Scorpius-Centaurus OB association. Spectra of the field stars have been retrieved from the ESO archive of

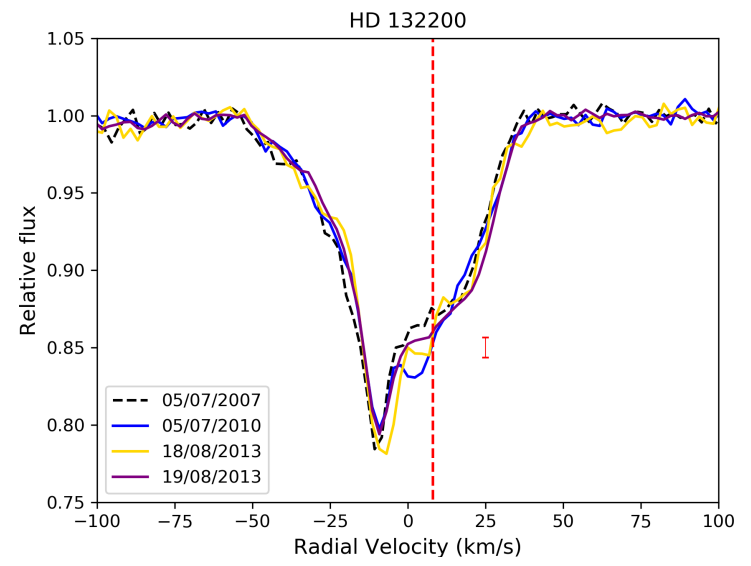

Fig. A.1. Ca II K line profiles of HD 132200. Solid lines are for FEROS spectra and the dashed line is for a HARPS spectrum. Observing dates are indicated. Vertical red dashed line indicates the radial velocity of the star. A $3 \sigma$ error bar is plotted in red.

FEROS $^{4}$, HARPS $^{5}$ and UVES ${ }^{6}$, as well as our own observations in the case of HD 146897. Field stars around each debris-disc star, their angular distances, stellar radial velocities, and velocities of non-photospheric features are given in Tables A.1 to A.4. HD 131488. The field stars HD 132058, HD 133937, and HD 135454 have features at $\sim-20 \mathrm{~km} \mathrm{~s}^{-1}$ (Table A.1), relatively close in velocity to the velocity vectors of the $\mathrm{G}$ and Mic clouds in the Colorado model. HD 128207, HD 131120, HD 132094, and HD 133880 have a common feature at $\sim-13 \mathrm{~km} \mathrm{~s}^{-1}$, suggesting it is an interstellar absorption not traced in the Colorado model. The star HD 132200 presents two features at $-6.9 \mathrm{~km} \mathrm{~s}^{-1}$ and $4.6 \mathrm{~km} \mathrm{~s}^{-1}$, which are close to the ones observed in HD 131488 at $-3.2 \mathrm{~km} \mathrm{~s}^{-1}$ and $4.0 \mathrm{~km} \mathrm{~s}^{-1}$. The HD 132200 feature at $6.9 \mathrm{~km} \mathrm{~s}^{-1}$ remains practically constant. This absorption, and the $-3.2 \mathrm{~km} \mathrm{~s}^{-1}$ of our debris-disc star, are close to the mean velocity of $\sim-6.6 \mathrm{~km} \mathrm{~s}^{-1}$ that characterize the approaching face of the bubble around the OB association (Pöppel et al. 2010). Thus, they could have an ISM origin. At the same time, the HD 132200 $4.6 \mathrm{~km} \mathrm{~s}^{-1}$ feature varies significantly (Fig. A.1), which suggests it is related to the star. This fact also suggests that the $4.0 \mathrm{~km} \mathrm{~s}^{-1}$ absorption in HD 131488, which coincides with the stellar radial velocity, is likely circumstellar.

$H D$ 138813. Like HD 138813, all field stars show a nonphotospheric absorption Na I D2 at $\sim-13 \mathrm{~km} \mathrm{~s}^{-1}$ (Table A.2), strongly suggesting an ISM origin.

HD 131835. All field stars, as well as HD 131835, share the $\sim-13.0 \mathrm{~km} \mathrm{~s}^{-1}$ feature mentioned above (Table A.3), which likely has an ISM origin. One of the field stars, HD 132955, presents a second, strong feature at $\sim 3.8 \mathrm{~km} \mathrm{~s}^{-1}$. Our star, HD 131835, also has a very weak (requiring confirmation) $2.2 \mathrm{~km} \mathrm{~s}^{-1}$ feature at the stellar radial velocity. If real, its CS or ISM origin is ambiguous. None of the field stars have a feature close to the one at $-5.9 \mathrm{~km} \mathrm{~s}^{-1}$ of HD 131835. This absorption might correspond to the one at $-6.6 \mathrm{~km} \mathrm{~s}^{-1}$ which characterizes the mentioned bubble of the $\mathrm{OB}$ association.

HD 146897. Absorption features at velocities close to the ones of HD 146897 are detected in the Ca II and/or Na I lines of the field stars (Table A.4). This suggests an ISM origin for the HD 146897 non-photospheric absorptions.

\footnotetext{
4 http://archive.eso.org/wdb/wdb/adp/phase3_spectral/ form?collection_name=FEROS

5 http://archive.eso.org/wdb/wdb/eso/repro/form

6 http://archive. eso.org/wdb/wdb/eso/uves/form
} 
Appendix B: Observing log

Table B.1. Observing Log.

\begin{tabular}{|c|c|c|c|c|c|c|}
\hline Star & Date (UT) & Instrument & Date (UT) & Instrument & Date (UT) & Instrument \\
\hline HD 9672 & 20150904T0107 & HERMES & $20150904 \mathrm{~T} 0143$ & HERMES & 20150904T0318 & HERMES \\
\hline \multirow[t]{12}{*}{ (49 Cet) } & $20150904 \mathrm{~T} 0539$ & HERMES & $20150905 \mathrm{~T} 0117$ & HERMES & 20150905 T0133 & HERMES \\
\hline & 20150905 T0335 & HERMES & 20150905 T0523 & HERMES & 20150906Т0126 & HERMES \\
\hline & $20150906 \mathrm{~T} 0221$ & HERMES & $20150906 \mathrm{~T} 0237$ & HERMES & 20150906T0529 & HERMES \\
\hline & $20150907 \mathrm{~T} 0113$ & HERMES & $20150907 T 0129$ & HERMES & $20150907 T 0414$ & HERMES \\
\hline & 20150907 T0430 & HERMES & 20151220 T2047 & HERMES & 20151220T2108 & HERMES \\
\hline & $20151222 \mathrm{~T} 2122$ & HERMES & 20151222T2148 & HERMES & $20151222 \mathrm{~T} 2213$ & HERMES \\
\hline & $20151223 \mathrm{~T} 2059$ & HERMES & $20151223 \mathrm{~T} 2230$ & HERMES & $20160126 \mathrm{~T} 2000$ & FIES \\
\hline & $20160126 \mathrm{~T} 2044$ & FIES & $20160127 \mathrm{~T} 1940$ & FIES & $20160127 \mathrm{~T} 1949$ & FIES \\
\hline & $20160127 \mathrm{~T} 1954$ & HERMES & $20160127 \mathrm{~T} 2006$ & HERMES & $20160128 \mathrm{~T} 1934$ & HERMES \\
\hline & $20160128 \mathrm{~T} 1946$ & HERMES & 20160130 T2004 & HERMES & 20160130 T2017 & HERMES \\
\hline & $20160714 \mathrm{~T} 0505$ & HERMES & 20160715 T0457 & HERMES & $20160717 \mathrm{~T} 0510$ & FIES \\
\hline & 20160718T0502 & FIES & 20160719 T0453 & FIES & & \\
\hline \multirow[t]{9}{*}{ HD 21997} & $20150904 T 0503$ & HERMES & $20150905 \mathrm{~T} 0422$ & HERMES & 20150905 T0443 & HERMES \\
\hline & 20150906T0448 & HERMES & 20150906Т0508 & HERMES & $20150907 \mathrm{~T} 0451$ & HERMES \\
\hline & $20150907 T 0512$ & HERMES & 20150919 T0453 & TIGRE & 20150920Т0251 & TIGRE \\
\hline & $20150924 \mathrm{~T} 0340$ & TIGRE & 20150925 T0311 & TIGRE & 20150926T0440 & TIGRE \\
\hline & $20151023 \mathrm{~T} 0225$ & FEROS & $20151024 T 0526$ & FEROS & 20151023Т0611 & FEROS \\
\hline & $20151110 \mathrm{~T} 2302$ & TIGRE & $20151111 \mathrm{~T} 2230$ & TIGRE & 20151114 T0058 & TIGRE \\
\hline & $20151114 \mathrm{~T} 2216$ & TIGRE & $20151220 \mathrm{~T} 2220$ & HERMES & $20151220 \mathrm{~T} 2251$ & HERMES \\
\hline & $20151223 \mathrm{~T} 2304$ & HERMES & $20160127 \mathrm{~T} 2030$ & HERMES & $20160128 T 2011$ & HERMES \\
\hline & $20160130 \mathrm{~T} 2050$ & HERMES & & & & \\
\hline \multirow[t]{7}{*}{ HD 32297} & 20150904T0405 & HERMES & $20150904 \mathrm{~T} 0436$ & HERMES & $20151221 \mathrm{~T} 0106$ & HERMES \\
\hline & $20151221 \mathrm{~T} 0137$ & HERMES & $20151221 \mathrm{~T} 0208$ & HERMES & $20151223 \mathrm{~T} 0050$ & HERMES \\
\hline & 20151223T0121 & HERMES & $20151224 \mathrm{~T} 0159$ & HERMES & $20151224 \mathrm{~T} 0230$ & HERMES \\
\hline & $20160126 \mathrm{~T} 2148$ & FIES & $20160126 \mathrm{~T} 2220$ & FIES & $20160127 \mathrm{~T} 2037$ & FIES \\
\hline & $20160127 T 2108$ & FIES & $20160127 \mathrm{~T} 2231$ & HERMES & $20160127 \mathrm{~T} 2301$ & HERMES \\
\hline & $20160128 \mathrm{~T} 2204$ & HERMES & $20160128 \mathrm{~T} 2235$ & HERMES & $20160130 \mathrm{~T} 2231$ & HERMES \\
\hline & $20160130 \mathrm{~T} 2302$ & HERMES & & & & \\
\hline $\begin{array}{l}\text { HD } 109085 \\
(\eta \text { Crv })\end{array}$ & 20151221 T0642 & HERMES & 20160129T0528 & HERMES & $20160131 \mathrm{~T} 0305$ & HERMES \\
\hline \multirow{3}{*}{ (HIP 61782) } & 20170402 T0440 & FEROS & 20170403 T0414 & FEROS & 20170404T0402 & FEROS \\
\hline & 20170405 T0329 & FEROS & 20170406Т0331 & FEROS & $20170407 T 0418$ & FEROS \\
\hline & $20170408 T 0251$ & FEROS & 20170409 T0410 & FEROS & & \\
\hline HD 121191 & $20170601 \mathrm{~T} 0121$ & FEROS & & & & \\
\hline HD 121617 & 20170409T0238 & FEROS & 20170409T0611 & FEROS & & \\
\hline HD 131488 & 20170409T0219 & FEROS & 20170409T0551 & FEROS & & \\
\hline \multirow{5}{*}{$\begin{array}{l}\text { HD } 131835 \\
\text { (HIP } 73145)\end{array}$} & 20160326T0408 & FEROS & 20160326T0755 & FEROS & $20160327 \mathrm{~T} 0322$ & FEROS \\
\hline & $20160327 T 0544$ & FEROS & $20160327 T 0754$ & FEROS & $20160328 T 0315$ & FEROS \\
\hline & $20160328 \mathrm{~T} 0455$ & FEROS & 20160328T0808 & FEROS & 20160329T0304 & FEROS \\
\hline & 20160329T0429 & FEROS & 20160329T0836 & FEROS & 20170403 T0519 & FEROS \\
\hline & $20170407 \mathrm{~T} 0456$ & FEROS & $20170408 \mathrm{~T} 0322$ & FEROS & & \\
\hline HD 138813 & $20160304 T 0412$ & HERMES & $20160304 T 0443$ & HERMES & 20160305 T0407 & HERMES \\
\hline \multirow{12}{*}{ (HIP 76310) } & 20160305T0438 & HERMES & 20160305 T0509 & HERMES & 20160306T0418 & HERMES \\
\hline & 20160306T0449 & HERMES & $20160326 \mathrm{~T} 0424$ & FEROS & 20160326 T0817 & FEROS \\
\hline & $20160327 \mathrm{~T} 0336$ & FEROS & $20160327 \mathrm{~T} 0626$ & FEROS & 20160327T0828 & FEROS \\
\hline & $20160328 T 0421$ & FEROS & $20160328 \mathrm{~T} 0833$ & FEROS & 20160329T0506 & FEROS \\
\hline & 20160329T0901 & FEROS & $20160711 \mathrm{~T} 2142$ & HERMES & $20160712 \mathrm{~T} 2233$ & HERMES \\
\hline & $20160712 \mathrm{~T} 2304$ & HERMES & $20160713 \mathrm{~T} 2148$ & HERMES & $20160713 \mathrm{~T} 2219$ & HERMES \\
\hline & $20160714 \mathrm{~T} 2122$ & HERMES & 20160716T2128 & FIES & $20160717 T 2148$ & FIES \\
\hline & $20160718 \mathrm{~T} 2202$ & FIES & $20160719 T 2230$ & FIES & 20170309Т0518 & HERMES \\
\hline & 20170310 T0610 & HERMES & 20170310T0639 & HERMES & $20170314 \mathrm{~T} 0452$ & HERMES \\
\hline & $20170314 \mathrm{~T} 0523$ & HERMES & 20170314 T0554 & HERMES & 20170403 T0452 & FEROS \\
\hline & $20170404 \mathrm{~T} 0421$ & HERMES & 20170405 T0505 & FEROS & 20170406 T0443 & FEROS \\
\hline & $20170408 \mathrm{~T} 0354$ & FEROS & & & & \\
\hline
\end{tabular}

Notes. Columns are: Stars, Dates (Universal Time) and Instruments. 
Table B.1. continued.

\begin{tabular}{|c|c|c|c|c|c|c|}
\hline Star & Date (UT) & Instrument & Date (UT) & Instrument & Date (UT) & Instrument \\
\hline HD 146897 & $20160712 \mathrm{~T} 2115$ & HERMES & $20160712 T 2146$ & HERMES & 20170405T0702 & FEROS \\
\hline (HIP 79977) & 20170405 T0733 & FEROS & 20170407 T0726 & FEROS & 20170407T0756 & FEROS \\
\hline HD 156623 & $20151022 \mathrm{~T} 0514$ & FEROS & $20160328 \mathrm{~T} 0520$ & FEROS & 20170405 T0839 & FEROS \\
\hline \multirow[t]{4}{*}{ (HIP 84881) } & 20160326T0438 & FEROS & 20160328T0858 & FEROS & 20170406 T0619 & FEROS \\
\hline & 20160326T0838 & FEROS & 20160329T0655 & FEROS & $20170408 T 0717$ & FEROS \\
\hline & $20160327 \mathrm{~T} 0441$ & FEROS & 20160329T0925 & FEROS & & \\
\hline & 20160327T0902 & FEROS & 20170402 T0931 & FEROS & & \\
\hline \multirow[t]{6}{*}{ HD 172555} & $20151022 \mathrm{~T} 0356$ & FEROS & $20151023 \mathrm{~T} 0238$ & FEROS & 20160326 T0534 & FEROS \\
\hline & $20160326 T 0849$ & FEROS & $20160327 \mathrm{~T} 0715$ & FEROS & 20160327T0958 & FEROS \\
\hline & $20160328 T 0638$ & FEROS & $20160328 T 0943$ & FEROS & 20160329T0706 & FEROS \\
\hline & 20160329T0934 & FEROS & 20170402 T0629 & FEROS & 20170403Т0721 & FEROS \\
\hline & 20170404T0707 & FEROS & 20170405 T0657 & FEROS & 20170406T0703 & FEROS \\
\hline & 20170407 T0656 & FEROS & $20170408 \mathrm{~T} 0647$ & FEROS & & \\
\hline \multirow{6}{*}{$\begin{array}{l}\text { HD } 181296 \\
(\eta \text { Tel })\end{array}$} & $20151022 \mathrm{~T} 0357$ & FEROS & $20151023 \mathrm{~T} 0041$ & FEROS & 20151024T0309 & FEROS \\
\hline & 20160326T0620 & FEROS & $20160327 \mathrm{~T} 0719$ & FEROS & $20160327 \mathrm{~T} 1004$ & FEROS \\
\hline & $20160328 T 0644$ & FEROS & $20160328 T 0949$ & FEROS & 20160329T0712 & FEROS \\
\hline & 20160329T0942 & FEROS & 20170403 T0726 & FEROS & $20170404 \mathrm{~T} 0739$ & FEROS \\
\hline & $20170405 T 0815$ & FEROS & 20170406T0802 & FEROS & 20170407T0840 & FEROS \\
\hline & 20170408T0652 & FEROS & & & & \\
\hline \multirow[t]{2}{*}{ HD 181327} & 20170403T0731 & FEROS & $20170404 T 0713$ & FEROS & 20170406T0806 & FEROS \\
\hline & $20170407 \mathrm{~T} 0845$ & FEROS & $20170408 T 0657$ & FEROS & & \\
\hline
\end{tabular}

